\title{
Research Paper \\ Relationship Between Comorbidity, Chronic Diseases, ICU Hospitalization, and Death Rate in the Elderly With Coronavirus Infection
}

\author{
Hamed Akhavizadegan ${ }^{1,2}\left({ }^{\circ}\right.$, Mahmood Aghaziarati ${ }^{1} \odot{ }^{\circ}$, Mohammad Ghasem Roshanfekr Balalemi ${ }^{1}$, Zahra Arman Brou-

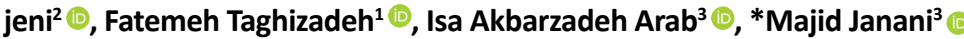

1. Department of Urology, Baharloo Hospital, Tehran University of Medical Sciences, Tehran, Iran.

2. Department of Research, Baharloo Hospital, Tehran University of Medical Sciences, Tehran, Iran.

3. Department of Epidemiology and Biostatistics, School of Health, Tehran University of Medical Sciences, Tehran, Iran.

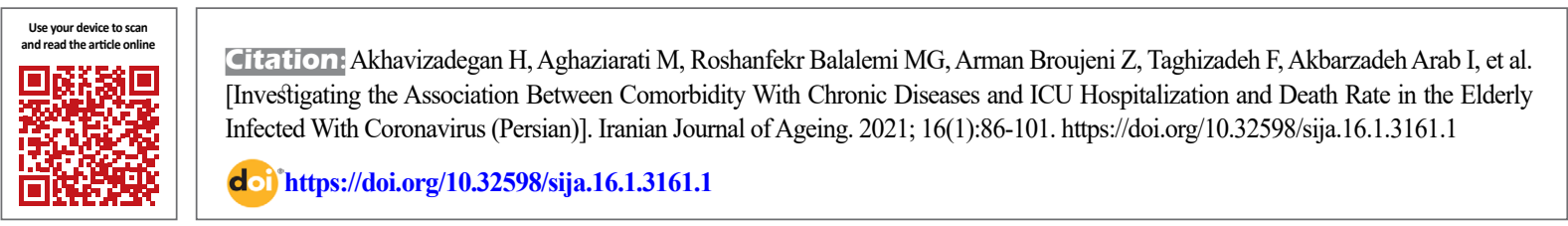

\section{(i) (8)}

Received: 08 Jan 2021

Accepted: 12 Mar 2021 Available Online: 01 Apr 2021

Key words: Comorbidity, Elderly, Coronavirus Disease 2019 (COVID-19) Mortality, Fatality

\section{A B STRACT}

Objectives The association of comorbidity diseases, such as diabetes and hypertension with the severity of Coronavirus Disease 2019 (COVID-19) has been repeatedly assessed. However, less research has specifically addressed the elderly. This study aimed to describe the demographic and clinical characteristics and evaluate the relationship between comorbidities, and the rates of Intensive Care Unit (ICU) hospitalization and death due to COVID-19 in the elderly.

Methods \& Materials This retrospective study was conducted in elderly patients with COVID-19 admitted to Baharloo Hospital. Demographic information, types of underlying diseases, and need for hospitalization in the ICU or death in hospital among the hospitalized patients from March 2020 to July 2020 was extracted from the COVID-19 hospital database. The logistic regression model was used to estimate the Odds Ratio (OR), as a separate measure of the association between the research variables' outcomes (death \& hospitalization outcomes in the ICU).

Results A total of 522 elderly patients were included in the study. Approximately $77 \%(n=422)$ of the elderly survived and $23 \%(n=100)$ died. The study participants' mean $\pm S D$ age was $72.55 \pm 8.44$ years. besides, and about 54\%( $n=281)$ were males. Logistic regression model results suggested that the odds of death in the study participants with $\geq 2$ underlying diseases equaled 1.69 (OR=1.69, 95\% $\mathrm{Cl}: 0.97-2.91, \mathrm{P}=0.04)$. Moreover, the odds of ICU hospitalization in them was $2.26(\mathrm{OR}=2.26,95 \% \mathrm{Cl}: 1.34-3.81, \mathrm{P}=0.002)$ time higher than their counterparts who did not have underlying diseases, i.e., statistically significant.

Conclusion Our results are expected to impact preventive interventions and take a more targeted approach to prioritize older patients with risk factors, rather than adopting calendar age policies as a general indicator for risk assessment.

\footnotetext{
Extended Abstract

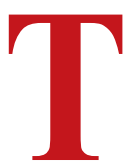

ered a pandemic $[1,2]$. Numerous deaths occurred globally due to this disease [3]. Although the COVID-19 is fatal in all ages, it is much severe in elderly patients $[4,5]$.

he rapid spread of Coronavirus Disease

Aging is among the critical risk factors for increased dis2019 (COVID-19) worldwide was considease severity and mortality in patients with COVID-19 [6].

\section{${ }^{*}$ Corresponding Author:}

Majid Janani

Address: Department of Epidemiology and Biostatistics, School of Health, Tehran University of Medical Sciences, Tehran, Iran

Tel: +98 (99) 81461046

E-mail: majid.jananiii@gmail.com
} 
Comorbidity of a condition with chronic diseases in the elderly is a common health problem worldwide [7], especially in countries with higher life expectancy $[8,9]$.

Triage, patient prioritization, and reducing COVID19-induced mortality are major challenges; thus, numerous studies have focused on the risk factors associated with COVID-19 outcomes in patients; however, data in elderly patients remains limited. Therefore, this study was performed to describe the demographic and clinical characteristics and to investigate the relationship between comorbidity and COVID-19 outcomes in the elderly.

\section{Materials and Methods}

This retrospective cohort study was conducted in patients aged $\geq 60$ years s with COVID-19, hospitalized in Baharloo Hospital. All included patients were diagnosed with COVID-19 as per a positive Polymerase Chain Reaction (PCR) test, a typical view of COVID-19on Computer Tomography (CT) scan, or radiography data.

The research participants' demographic data, including age, gender, Body Mass Index (BMI), disease severity, comorbidity with chronic diseases, and outcome variables, including the duration of hospitalization, a history of Intensive Care Unit (ICU) hospitalization, and disease outcome (death/alive) were collected from the admission records.

An Independent Samples t-test was used to compare the mean scores of the quantitative data between the dead and surviving groups. Besides, the Chi-squared test was used to investigate the relationship between qualitative variables and the death or survival of the explored patients. Analysis of Variance (ANOVA) was also used to compare the mean values of the quantitative variables in groups with one, $\geq 2$, and without comorbidities. The logistic regression model was used to investigate the relationship between comorbidity and outcomes. The Kaplan-Meier and Logrank tests were used to report survival and compare survival in the elderly with/without underlying disease. STATA was used for data analysis; the significance level of $<0.05$ was bilaterally considered in all analyzes.

\section{Results}

Overall, 522 elderly patients admitted to Baharloo Hospital were included in the present study. Approximately

Table 1. The demographic characteristics and symptoms of the elderly with COVID-19 at the time of hospitalization by underlying disease subgroups

\begin{tabular}{|c|c|c|c|c|c|}
\hline \multirow[b]{2}{*}{ Characteristic } & \multicolumn{4}{|c|}{ Mean士SD/ No. (\%) } & \multirow[b]{2}{*}{$\mathbf{P}$} \\
\hline & Total $(n=522)$ & $\begin{array}{l}\text { Without Comorbidity } \\
\qquad(\mathrm{n}=152)\end{array}$ & $\begin{array}{l}\text { With One Underlying } \\
\text { Disease }(n=157)\end{array}$ & $\begin{array}{l}\text { With two or More Under- } \\
\text { lying Diseases }(n=213)\end{array}$ & \\
\hline Age & $72.55 \pm 8.44$ & $70.30 \pm 8.46$ & $73.43 \pm 8.76$ & $73.49 \pm 7.90$ & $<0.001$ \\
\hline $\mathrm{BMI}, \mathrm{kg} / \mathrm{m}^{2}$ & $26.56 \pm 4.21$ & $26.52 \pm 3.74$ & $25.58 \pm 4.54$ & $27.01 \pm 4.13$ & 0.067 \\
\hline Gender (male) & $281(53.8)$ & $87(57.2)$ & $87(55.4)$ & $107(50.2)$ & 0.372 \\
\hline female & $241(46.2)$ & $65(42.8)$ & $70(44.6)$ & $106(49.8)$ & \\
\hline \multicolumn{6}{|l|}{ Symptoms } \\
\hline Fever & $267(51.1)$ & $71(46.7)$ & $90(57.3)$ & $106(49.8)$ & 0.153 \\
\hline Chills & $144(27.6)$ & 30 (19.7) & $51(32.5)$ & $63(29.6)$ & 0.030 \\
\hline Myalgia & $138(26.4)$ & $36(23.7)$ & $42(26.8)$ & $60(28.2)$ & 0.629 \\
\hline Cough & $319(61.1)$ & $106(69.7)$ & $86(54.8)$ & $127(59.6)$ & 0.022 \\
\hline Dyspnea & $363(69.5)$ & $94(61.8)$ & $111(70.7)$ & $158(74.2)$ & 0.038 \\
\hline $\begin{array}{l}\text { Hospitalization dura- } \\
\text { tion (days)* }\end{array}$ & $6(6.7)$ & $4.5(8)$ & $6(7)$ & $7(5.5)$ & 0.007 \\
\hline ICU admission & $147(28.2)$ & $26(17.1)$ & $46(29.3)$ & $75(35.2)$ & $<0.001$ \\
\hline Death & $121(23.2)$ & $24(15.8)$ & 39 (24.8) & $58(27.2)$ & 0.032 \\
\hline
\end{tabular}


Table 2. The ORs of underlying diseases by each disease and the combination of underlying diseases to cause death or hospitalization

\begin{tabular}{ccccc}
\hline \multirow{2}{*}{ Comorbidity } & \multicolumn{2}{c}{ Outcome: Death } & \multicolumn{1}{c}{ Outcome: ICU Hospitalization } \\
\cline { 2 - 5 } & OR $(95 \% \mathrm{Cl})$ & $\mathbf{P}$ & OR (95\%Cl) & P \\
\hline Cardiovascular disease & $1.07(1.04-1.09)$ & $<0.001$ & $1.64(1.05-2.55)$ & 0.027 \\
Respiratory diseases & $0.72(0.24-1.55)$ & 0.303 & $1.32(0.61-2.84)$ & 0.303 \\
\hline Renal Diseases & $1.23(0.48-3.13)$ & 0.651 & $1.70(0.71-4.05)$ & - \\
\hline Hypertension & $1.21(0.79-3.13)$ & 0.373 & $1.41(0.95-2.10)$ & 0.86 \\
\hline Diabetes & $1.30(0.85-2.01)$ & 0.222 & $1.70(1.13-2.56)$ & 0.10 \\
\hline Stroke (brain) & $0.90(0.45-1.18)$ & 0.780 & $2.13(1.14-3.97)$ & 0.017 \\
\hline Thyroid disease & $0.89(0.22-2.38)$ & 0.852 & $1.48(0.45-4.83)$ & 0.516 \\
\hline Rheumatic diseases & $2.14(0.75-8.23)$ & 0.136 & $1.26(0.36-4.41)$ & 0.709 \\
\hline Without underlying disease & 1 & - & 1 & - \\
\hline With one underlying disease & $1.46(0.82-2.63)$ & 0.199 & $1.68(0.95-2.95)$ & 0.071 \\
\hline With two or more underlying diseases & $1.69(0.97-2.91)$ & 0.040 & $2.26(1.34-3.81)$ & 0.002 \\
\hline
\end{tabular}

$77 \%$ ( $n=422)$ of the hospitalized elderly survived the disease, and $23 \%$ died. The Mean \pm SD age of the study participants was $72.55 \pm 8.44$ years, and about $54 \%(281)$ of them were males. The age of the elderly who expired due to COVID-19 (76.31 \pm 8.66 y) was older than that in the surviving elderly (71.41 \pm 08.04 y), i.e., statistically significant $(\mathrm{P}<0.001)$ (Table 1).

Furthermore, a significantly higher proportion of the expired elderly patients had cardiovascular diseases, compared to the rest $(\mathrm{P}=0.011)$. Additionally, the mortality rate in the elderly with one or more underlying diseases was significantly higher than that in the elderly without such conditions $(24.8 \%$ vs. $15.8 \% ; \mathrm{P}=0.032)$ (Table 2$)$.

Logistic regression analysis suggested that having cardiovascular disease increases the odds of death in the elderly by 1.07 (OR=1.07, 95\%CI: 1.04-1.09); also, the odds of death in participants who had $\geq 2$ underlying diseases was 1.69 (OR=1.69, 95\%CI: 0.97-2.91, $\mathrm{P}=0.04)$ times higher than that in the elderly without such conditions, i.e., significant (Table 2). Moreover, the regression results for each outcome are presented in Table 2.

As shown in Figure 1, the survival rate of the patients without underlying diseases was higher than that in the patients with underlying diseases (Figure 1B). Besides, the frequencies of death and ICU hospitalization were directly related to the underlying diseases. Additionally, the elderly with a higher frequency of underlying diseases generated a higher mortality rate and ICU hospitalization (Figure 1C).

\section{Discussion and Conclusion}

This study described the demographic and clinical characteristics and investigated the association between underlying diseases and the severity of COVID-19 in the elderly. Numerous studies suggested that the mortality rate in the elderly with COVID-19is much higher than that in the young population $[4,5,10]$. The obtained results also signified that approximately $23 \%$ of the elderly with COVID-19 expired, i.e., higher than the mortality rate in China, Korea, and Italy $[4,11]$.

Patients with COVID-19 usually die from various causes, including multiple organ failure, shock, respiratory failure, heart failure, arrhythmias, and renal failure $[12,13]$. Previous studies indicated that a higher age can cause an inadequate response of the immune system to pathogens, the dysfunction of organs [14-17], and accelerated inflammation; eventually leading to multiple organ failure and death, and death in the ICU $[18,19]$.

Previous studies reported that comorbidity with other diseases may lead to a weakened immune system and 

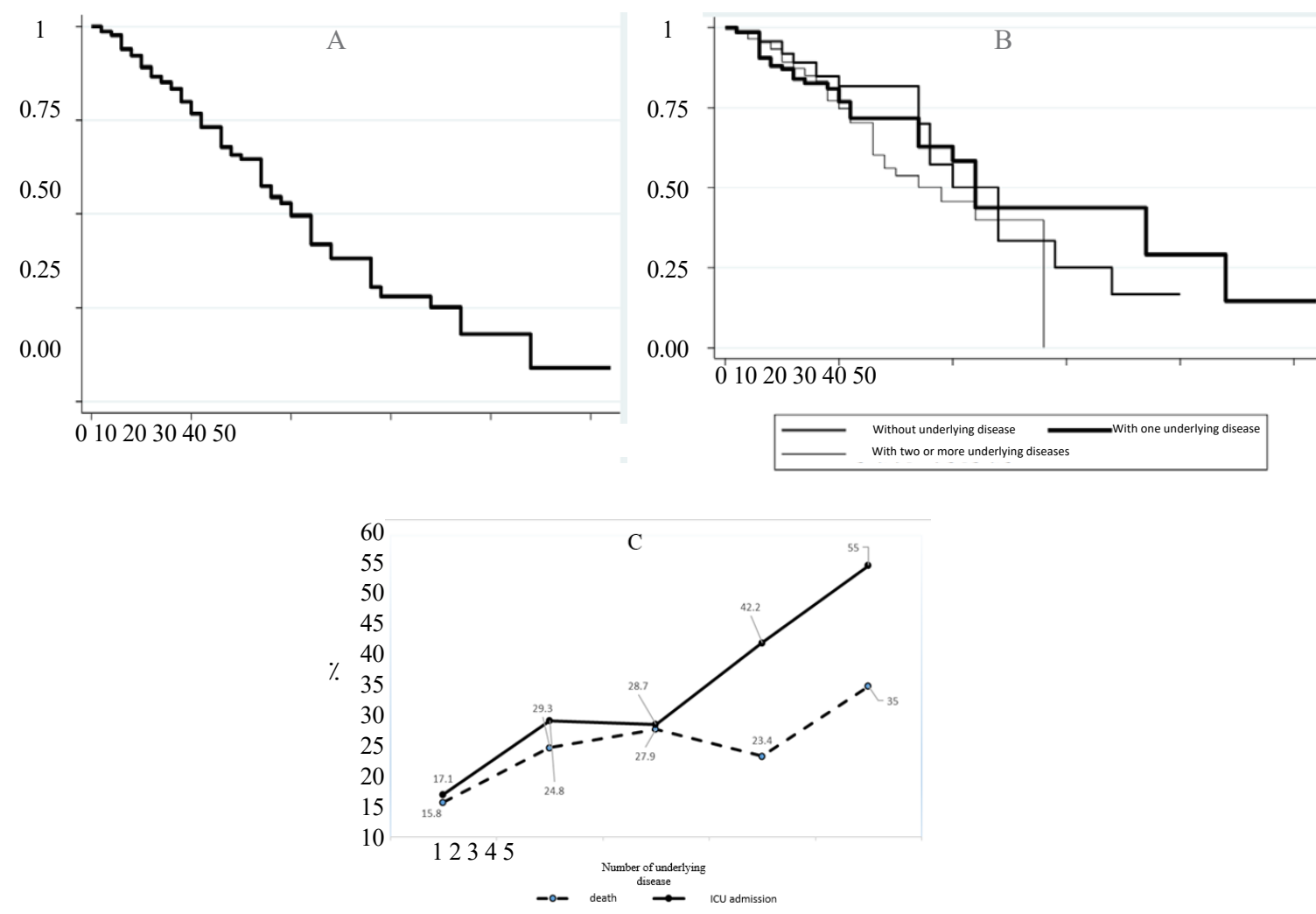

SALMAND

Figure 1. Kaplan-Meier survival odds chart in the studied elderly studied

A: in general; B: underlying diseases subgroup, and C: shows the association between the number of underlying diseases and death/ ICU admission.

the dysfunction of the body; thus, this condition presents higher adverse impacts in the elderly patient, compared to the young patients with COVID-19 [16, 17, 20, 21]. Previous studies, specified cardiovascular disease, obstructive pulmonary disease, hypertension, and diabetes as the most critical risk factors for the severity and mortality of $\mathrm{CO}$ VID-19 [22].

Sun et al. examined 3400 patients and reported similar results to ours [45]. Ruan et al. also explored 150 patients with COVID-19 and concluded that cardiovascular disease and hypertension were higher in patients who expired due to the disease, compared to the patients who were discharged (43\% vs. $19 \%$; $\mathrm{P}<0.001$ ) [46]. A systematic review and meta-analysis study included 16 studies and 3994 patients; accordingly, the relevant data demonstrated that hypertension $(\mathrm{OR}=2.95)$, diabetes $(\mathrm{OR}=3.07)$, cardiovascular disease $(\mathrm{OR}=4.58)$, and chronic kidney disease $(\mathrm{OR}=5.32)$ generated a higher risk of mortality or undesirable outcomes in this group [47].

Finally, our results suggested that mortality is very high in the elderly with COVID-19. Chronic disease aggravates the prognosis in the elderly. Our results are expected to impact preventive interventions and take a more appropriate approach to prioritize older patients with risk factors, rather than adopting calendar age policies as a general indicator of risk. Furthermore, it seems that more care should be provided for the elderly with COVID-19 and underlying disease.

\section{Ethical Considerations}

\section{Compliance with ethical guidelines}

This study was approved by the Ethics Committee of the Tehran University of Medical Sciences (Code: IR.TUMS. VCR.REC.1399.148). All ethical principles are considered in this article. The participants were informed about the purpose of the research and its implementation stages. They were also assured about the confidentiality of their information and were free to leave the study whenever they wished, and if desired, the research results would be available to them. 


\section{Funding}

This study was performed using the recorded data of the patients admitted to Baharloo Hospital and supported by the Tehran University of Medical Sciences.

\section{Authors' contributions}

Research of texts: Hamed Akhavizadegan and Mohammad Ghasem Roshanfekr Balalemi; Drafting of the article: Hamed Akhovizadegan, Mahmoud Aghaziarti and Zahra Boroujeni; Completion and correction of the draft article: Majid Janani and Fatemeh Taghizadeh; Data analysis: Issa Akbarzadeh Arab and Majid Janani; Editing, completion and final approval: All authors.

\section{Conflicts of interest}

The authors declared no conflict of interest.

\section{Acknowledgements}

We would like to thank the staff and management of Baharloo Hospital who cooperated with us in the implementation of this project. 


\section{بررسى ارتباط ابتلاى همزمان بله بيمارىهاى زمينهاى و درصد بسترى در آى بسىيو و مرتى در

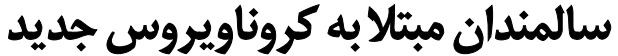

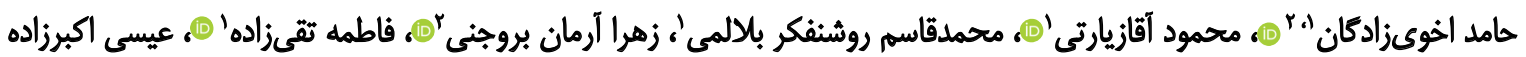

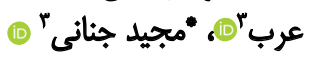

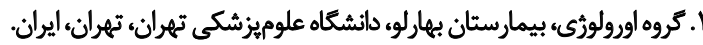

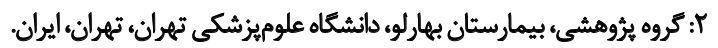

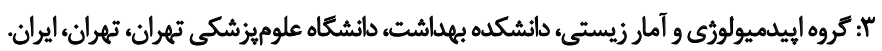

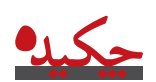

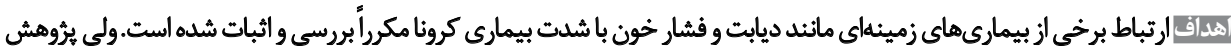

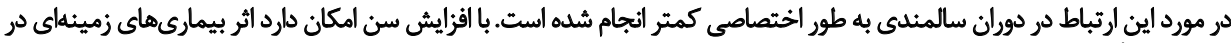

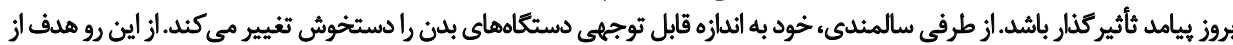

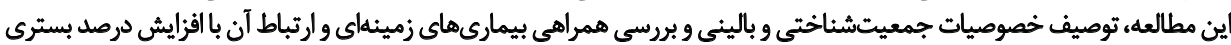

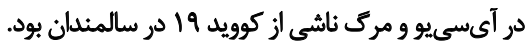

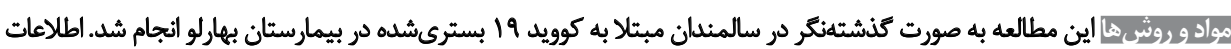

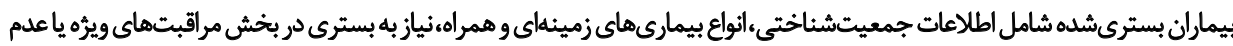

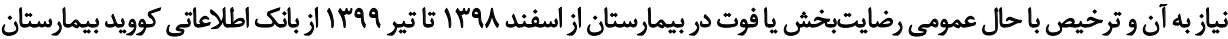

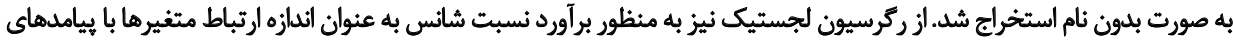

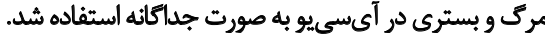

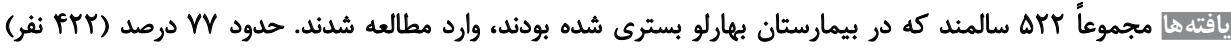

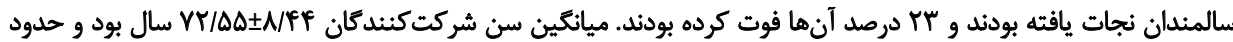

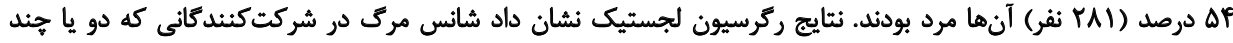

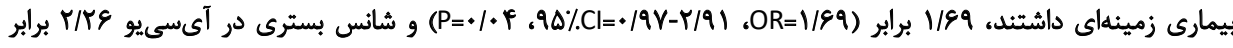

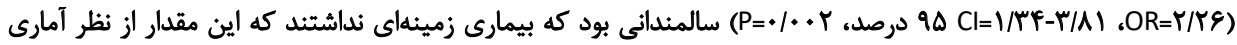

معنى دار بود.

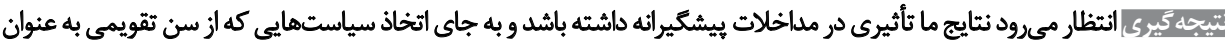

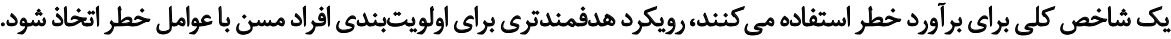

تاريخ دريافت: 19 دى 199

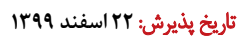

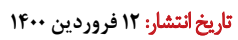

كليدوازوها:

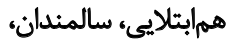

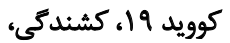
ميرايى كوويد

دادهاند ريسكفاكتورهاى مختلفى با شدت و كشند إنى كوريد

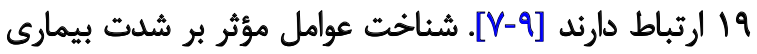

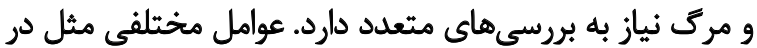

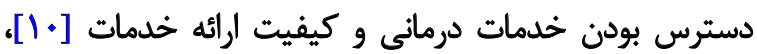

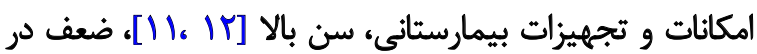

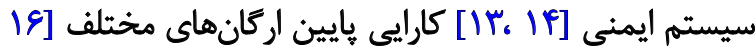

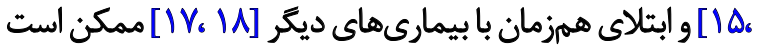

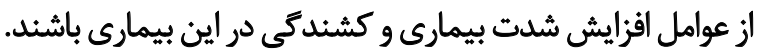

سالمندى به معناى سن بالاتر از .9 سال، يكى از

مقدمه

كروناويروس، عامل بيمارى سندروم حاد تنفسى، در دسامبر

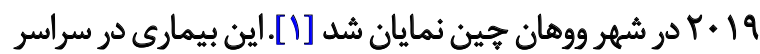

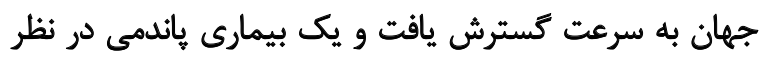

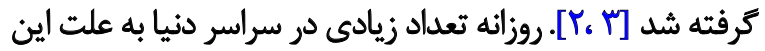

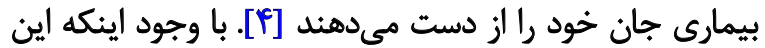

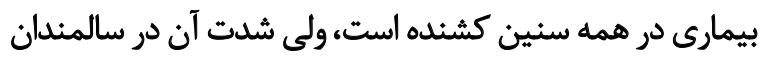

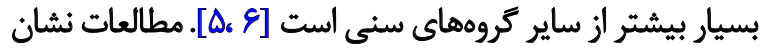

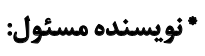

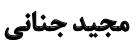
نشائي: تهران، دانشكاه علوميزشكى تهيران، دانشكده بهيداشت، كروه إيدميولوزي و آمار زيستى.

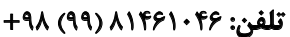
majid.jananiii@gmail.com : بلكت الكترونيكي: 
خون، ديابت، سكته مغزى، بيمارىهاى تيروئيدى و بيمارىهاى

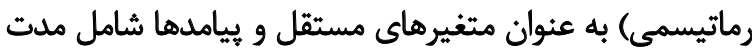

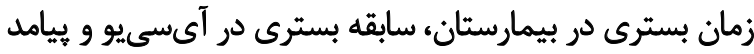

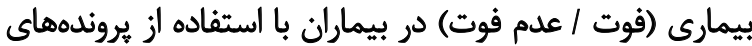
يزشكى جمع آورى شدند. آناليز آمارى

از ميانگين و انحراف معيار براى توصيف دادهایى كمى و از

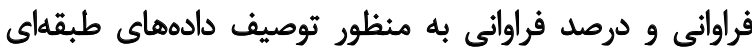

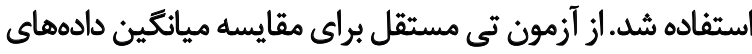

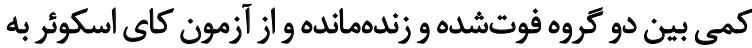

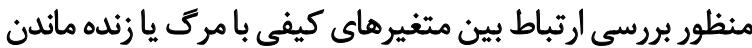

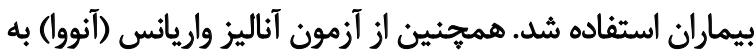

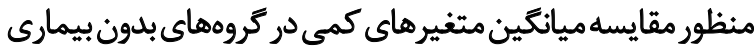

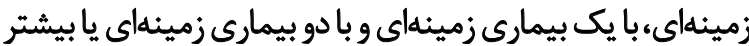

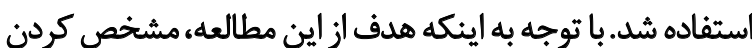

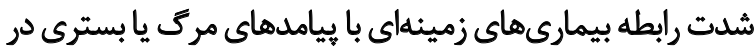

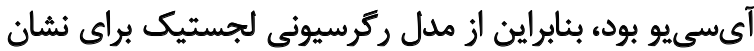

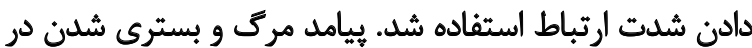

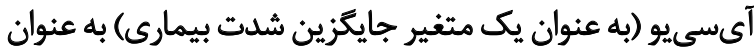

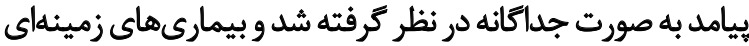
به عنوان مواجهه در مدل وارد شدند.

براى بررسى ارتباط بيمارىهاى زمينهاى با بيامدهاى مورد

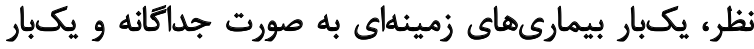

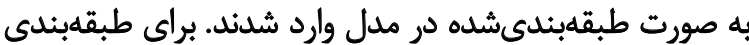

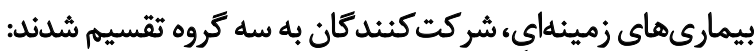

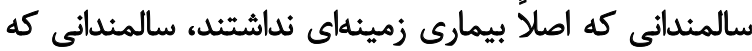

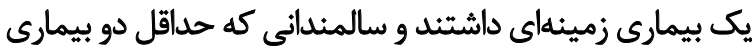

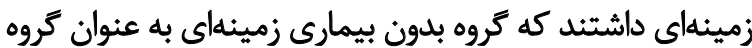

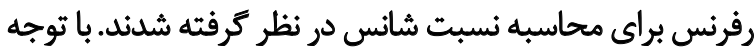

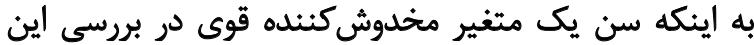

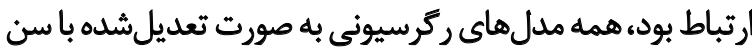

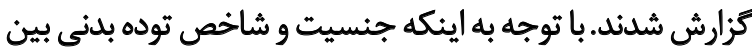

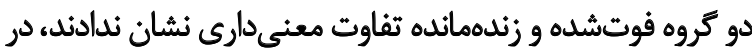
مدل ركرسيون به عنوان مخدوش زمر وارد نشدند.

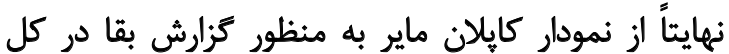

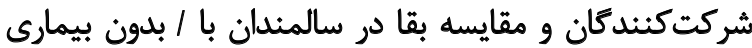

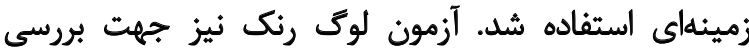

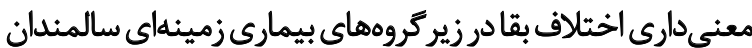

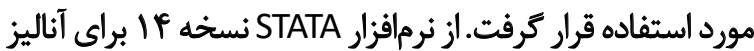

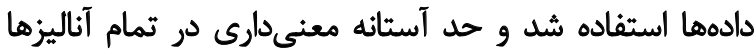

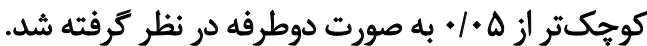

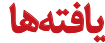

ريسكفاكتورهاى مهم افزايش شدت بيمارى و كشندئى در

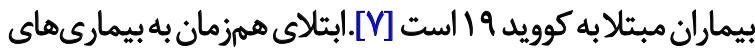

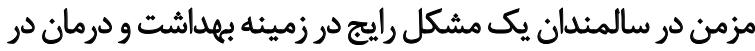

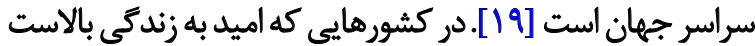

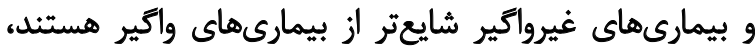

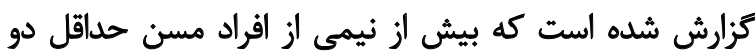

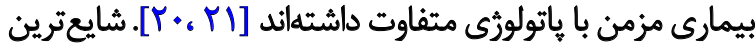

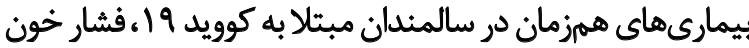

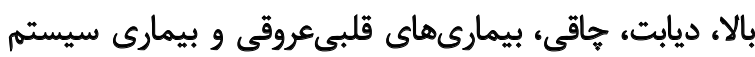

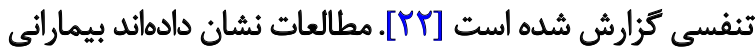

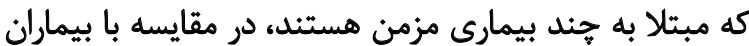

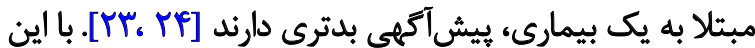

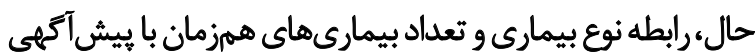

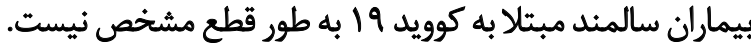

با توجه به آسيبذيذيرى سالمندان، بيشكيرى از شديد شدن

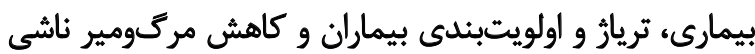

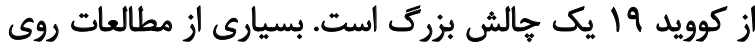

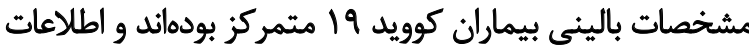

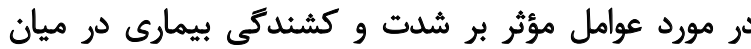

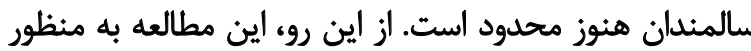

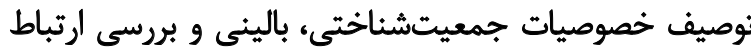

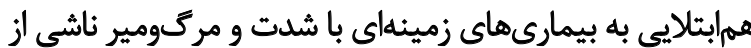

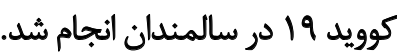
روش مطالعه - - ماله شركت كنئدَّان

اين مطالعه به صورت هم كروهى كذشتنهنكر' در سالمندان

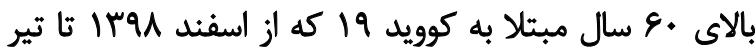

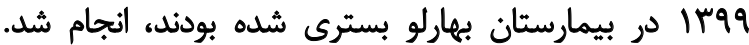

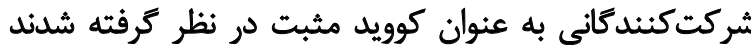

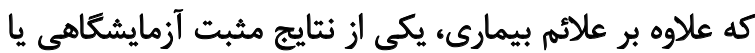

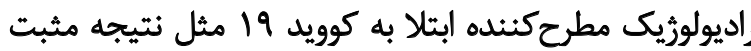

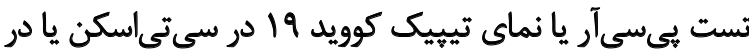

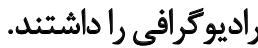
جمعآورى دادها اطلاعات جمعيتشناختى و زمينهاى شركت كنينداًن شامل

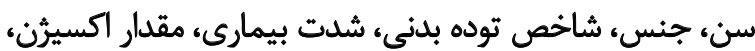

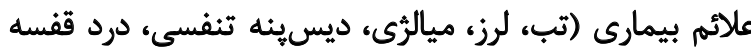

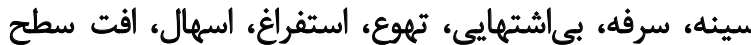

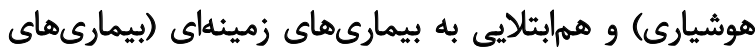

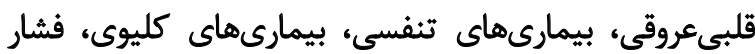




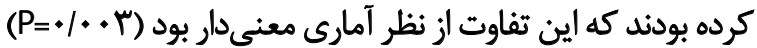

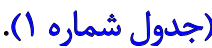

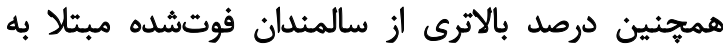

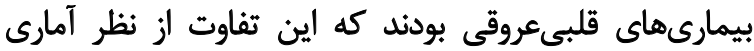

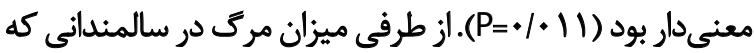

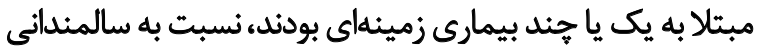

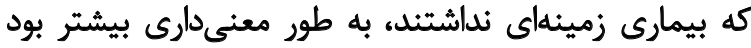
(ج=./. Tr $)$

نتايج نشان داد سن سالمندائى كه بيمارى زمينهاي داشتند

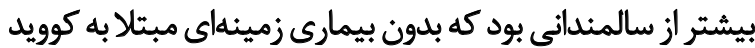

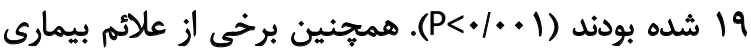

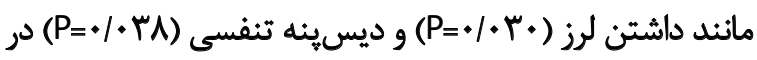

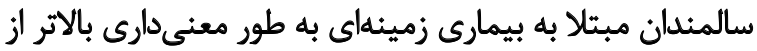

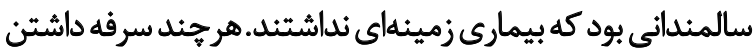

مجموعاً

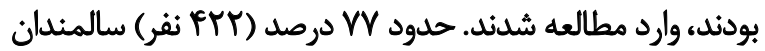

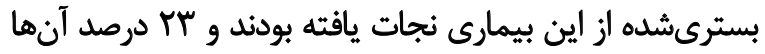

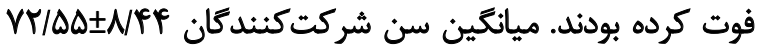

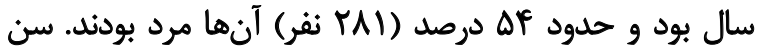
سالمندانى كه به دليل كوويد 19 فوت كرد

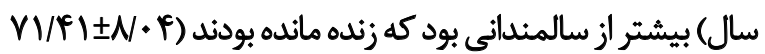

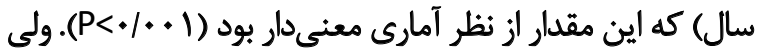

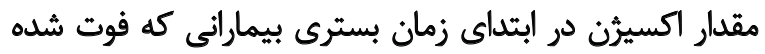

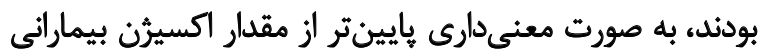

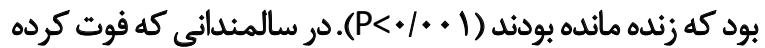

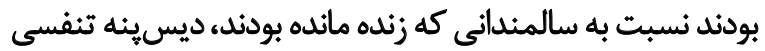
به

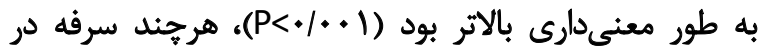
سالمنداني كه زنده مانده بودند بيشيتر از سالمنداني بود كه فوت

جدول ا. ويركى هاي جمعيتشناختي و علانم سالمندان مبتلا به كوويد 19 در بلدو بسترى در بيمارستان بهارلو به تفكيك موارد فوتشده و زنده

\begin{tabular}{|c|c|c|c|c|}
\hline \multirow[b]{2}{*}{ سطح معنى دارى } & \multicolumn{3}{|c|}{ ميانكيند|نحرافمعيار / تعداد (درصد) } & \multirow[b]{2}{*}{ متغيرها } \\
\hline & 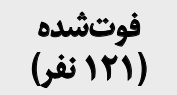 & زندهاندها & 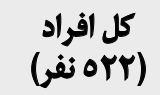 & \\
\hline $.1 \cdots 1$ & VE/MIIINEg & $V I / F^{e} \backslash \pm N \cdot f$ & $V T / \Delta \Delta \pm N / F$ & \multirow{4}{*}{ طبقلهبندي شيله } \\
\hline \multirow{3}{*}{.$\ldots 1$} & $\Delta q(f \cdot / 1)$ & $r \Delta \cdot(g g / V)$ & $r \cdot q(\Delta Q / Y)$ & \\
\hline & $\&)(f / \Delta)$ & $u(r / 1)$ & $109(r \cdot / 0)$ & \\
\hline & $\mathbb{W}\left(W^{N}\right)$ & $r V(V / T)$ & $\Delta f(1+/ \pi)$ & \\
\hline$+M r T$ & $r \& / 8 q \pm r / F \Delta$ & $r g / \Delta) \pm Y / \Delta$ & $r E / \Delta F \pm F / Y)$ & يانكين شاخص توده بلنى (كيلوكرم بر متر مريع) \\
\hline 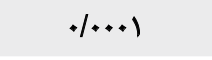 & $\Lambda F / K E \pm \mid r / \circ \Delta$ & 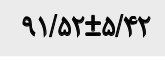 & AQ/VIINYF & ميانكين مقدار اكسيرن در بلو بسترى (درصد) \\
\hline \multirow{2}{*}{$+/ 1+r$} & $n(\Delta N Y)$ & $M T \cdot(\Delta T / T)$ & $r \wedge \mid(\Delta r / \Lambda)$ & \multirow{2}{*}{ 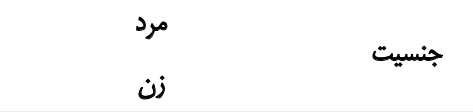 } \\
\hline & $\Delta \cdot(F / / T)$ & $19)(f Y / 8)$ & $M F I(F \& / M)$ & \\
\hline.$/ T 19$ & $\operatorname{Rv}(\Delta \Delta / F)$ & $r \cdot .(1 e q / q)$ & $\operatorname{rev}(\Delta / /)$ & ثب \\
\hline.$/ M^{e q}$ & $M(r \& / 8)$ & $M T(T V / q)$ & $\operatorname{lfF}(\mathrm{T} / \mathrm{P})$ & لز \\
\hline . & $\operatorname{me}($ YNI) & $1 . f(T \Delta / q)$ & $\operatorname{IHA}(T E / T)$ & 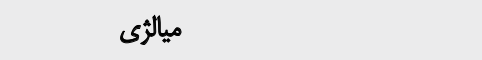 \\
\hline.$/ N F$ & • & $v(M / N)$ & $V(M / M)$ & آنوسمى \\
\hline $.1 .+4$ & $\operatorname{9r}(\lambda \cdot / T)$ & $r \& 8(89 / \pi)$ & rex (Eq/A) & ديسينه تنفسى \\
\hline . MAr & $q(V / \varphi)$ & $r \cdot(V / \Delta)$ & $r q(V / \Delta)$ & درد قفسه سينه \\
\hline.$/ .+r$ & $\varepsilon \cdot(p q / 8)$ & roq $(P P / \&)$ & r19 (EV/I) & علاتم بيمارى \\
\hline $.1+f$ & qf $(W / N)$ & $\Delta \Lambda(\mid f / \Delta)$ & $\Lambda \Delta(1 \notin / \pi)$ & بي أشتهايي \\
\hline - NGe & $M(I V / F)$ & $\varepsilon_{0}(1 \& / T)$ & $N \in(\mid \& / \Delta)$ & تهن \\
\hline +/rar & $17(1+N)$ & $M(N T)$ & $\forall \in(N A)$ & استفراغ \\
\hline.$/ \Delta F$ & $8(\Delta / \cdot)$ & $r \&(E / Q)$ & $M(\xi / 1)$ & أسهال \\
\hline$+\ldots 1$ & $m(19 / *)$ & $\Pi r(\% / \cdot)$ & $r \Delta(g / V)$ & افت سطح هوشيارى \\
\hline
\end{tabular}


جدول r. بيمارىهاى زميئهاى در سالمندان مبتلا به كوويد 19 به تفكيك موارد فوتشده و زئده

\begin{tabular}{|c|c|c|c|c|c|}
\hline معني سطارى & 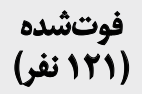 & زاندهاند & 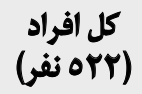 & \multicolumn{2}{|c|}{ مثغيرها } \\
\hline $.1+11$ & $r \cdot(m / 1)$ & $\operatorname{AY}(Y / V)$ & $\operatorname{Irr}(r \varphi / T)$ & بيمارى هاي قليى عروقي & \\
\hline . MAT & $\varepsilon(\Delta)$ & $r r(\varepsilon / V)$ & $M(\varepsilon / N)$ & بيمارى هاى تنفسى & \\
\hline.$/ 499$ & $V(\Delta / \Lambda)$ & $1 \varepsilon(\varphi)$ & $M(F / R)$ & بيمارىهاى كليوى & \\
\hline.$/ 194$ & ST (AT/N) & $\operatorname{Mr}(r \Delta / f)$ & $\operatorname{rfa}(f \& / 9)$ & فشار خون & \\
\hline.$/ \pi q$ & 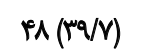 & IUA (MT/R) & $r N \in(r \Delta / 9)$ & ديابت & بيمارى هاى زمينهاى \\
\hline $.10 \Delta q$ & $\mathbb{1 r}(1 \cdot M)$ & $r q(9)$ & $r q(q / F)$ & سكته مغزى & \\
\hline.$/ 994$ & $r(T / \Delta)$ & $1 \cdot(Y / \Delta)$ & $M(T / \Delta)$ & ييمارىهاى تيروئيدى & \\
\hline.$/ 1 T a$ & $\Delta(\% / 1)$ & $V(1 / N)$ & $\mathbb{M}(T / M)$ & بيمارىهاى رماتيسمى & \\
\hline $.18 V 8$ & $1(* / A)$ & $r(\cdot / 0)$ & $r(\cdot / 8)$ & سرطان & \\
\hline \multirow{3}{*}{$.1 . \pi r$} & $r f(19 / 1)$ & $\operatorname{IrA}(\mathrm{TI} / q)$ & $\operatorname{lar}(Y Q /)$ & بدون بيمارى زمينهاى & \\
\hline & mq (m/T) & $111($ (T//) & $\operatorname{lar}(r \cdot /)$ & تنها با يك بيمارى زمينهاى & تعداد بيمارى هائ زمينهاي \\
\hline & $\Delta \wedge(P V / q)$ & $100(T N V)$ & $\operatorname{rir}(f \cdot / A)$ & با دو بيمارى زمينهاي يا بيشتر & \\
\hline
\end{tabular}

il

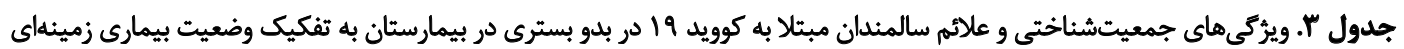

\begin{tabular}{|c|c|c|c|c|}
\hline \multirow{2}{*}{ معنيدارى سطع } & \multicolumn{3}{|c|}{ ميانكيندانحراف معيار / تعداد (درصد) } & \multirow[b]{2}{*}{ متغيرها } \\
\hline & 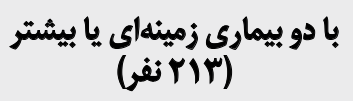 & 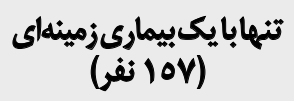 & 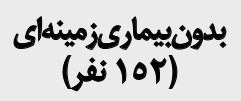 & \\
\hline $.1 . . .1$ & $V r / e q \pm V / q$. & $V T / R T \pm N V S$ & $V \cdot / r \cdot \pm N+8$ & هيانكين سنى (سال) \\
\hline $.1 .9 V$ & $r V / \cdot l \pm \varphi / / r$ & re/ALtP/AP & $r \Delta / \Delta Y \pm r / N^{C}$ & ميانكين شاخص توده بلدنى \\
\hline$+/ 19$ & MAY \pm IIV & $9 \cdot 1+1 \pm V / r+$ & Q./VA $\pm V / \& 8$ & ميانكين مقدار اكسيرن در بلو بسترى (درصد) \\
\hline \multirow{2}{*}{$\cdot / \pi T$} & $1 \cdot V(\Delta \cdot / \pi)$ & $\operatorname{AV}(\Delta \Delta / \mathcal{F})$ & $\operatorname{AV}(\Delta V / T)$ & مرد \\
\hline & $1.8(\varepsilon q / \lambda)$ & $V \cdot(f+18)$ & $g \Delta(F T / A)$ & جنسيك \\
\hline . / & $1.8(59 / 1)$ & $q_{*}(\Delta V / T)$ & $n(f \& / V)$ & تب \\
\hline .1 .4 & $\varepsilon r(r / g)$ & $\Delta)(M T / \Delta)$ & $r \cdot(19 / 7)$ & ليز \\
\hline . IqYq & E. (TNY) & $\operatorname{Pr}(\mathrm{r} / \mathrm{A})$ & Tre (MTM) & ميالثى \\
\hline.$/ T \Delta T$ & $\Delta\left(T / N^{\prime}\right)$ & $1(\cdot / 8)$ & $1(* / N)$ & آنوسمى - آن \\
\hline$+\%$ & $1 Q A(N / T)$ & $M(N+M)$ & if $(8 / / 1)$ & ديسينه تنفسى \\
\hline.$/ 4 Y 8$ & $M(q / q)$ & $9(\Delta / V)$ & $9(\Delta / 9)$ & درد قفسه سينه \\
\hline $.1 . M T$ & $\operatorname{IrV}(\Delta q / F)$ & $N(\Delta f / A)$ & $1.8(e q / V)$ & علاتم بيمارى \\
\hline.$/ 098$ & $M(I V / A)$ & $r E(18 / 9)$ & $M(M / A)$ & بيىاشتهايي \\
\hline $.1 \cdot r$ & $M(T \cdot M)$ & $r \&(18 \mid 8)$ & $I V(M / M)$ & تزק \\
\hline.$/+\lambda t$ & r) (q/q) & $M(1 / / \Delta)$ & $V(\varphi / 8)$ & استفراغ \\
\hline$+/ 1 V E$ & $\pi(\Delta / 9)$ & If (NQ) & $\varepsilon(\% / q)$ & اسبهال \\
\hline . / TrA & $19(N q)$ & $9(\Delta / V)$ & $V(\varphi / 8)$ & افت سطح هوشيارى \\
\hline
\end{tabular}


جدول F. يمامدهاي بيماران مبتلا به كوويد 19 در سالمندان بسترى به تفكيك وضعيت بيمارى زمينهاى

\begin{tabular}{|c|c|c|c|c|}
\hline \multirow[b]{2}{*}{ سطح معنى دارى } & \multicolumn{3}{|c|}{ تعداد (د,صد) } & \multirow[b]{2}{*}{ متغيرها } \\
\hline & با دو بيمارى زمينهاي يا بيشتر & 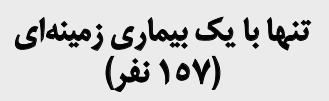 & 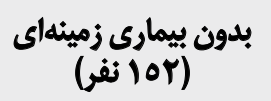 & \\
\hline $.1 \cdot . v$ & $V(\Delta / \Delta)$ & $8(V)$ & $r / \Delta(A)$ & هدتزمان بسترى" (روزها) \\
\hline $.1+.+1$ & $V \Delta(Y \Delta / Y)$ & $p e(r q /)$ & $r e(I V / N)$ & بسترى در آى سيىيو \\
\hline . & $\Delta \Lambda(T V / T)$ & $r q(T r / A)$ & $M P(I Q / \Lambda)$ & فوت \\
\hline
\end{tabular}

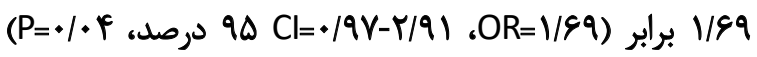
سالمندانى بود كه بيمارى زمينهاى نداشتند كه اين مقدار از نظر آمارى معنى دار بود (جدول شماره ه).

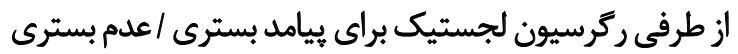

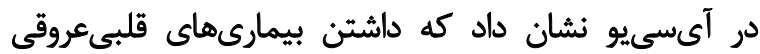

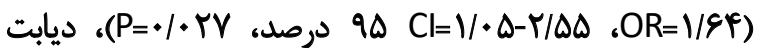

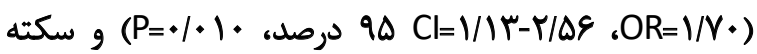

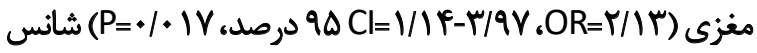
بسترى در آىسىيو را افزايش مي دهد. همجنين به صورت كلى

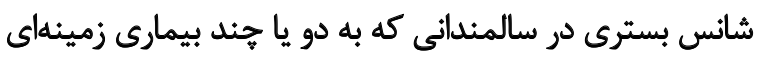

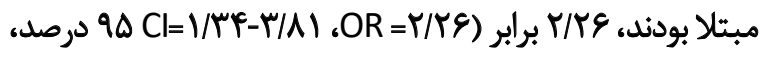

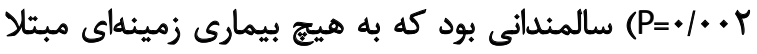

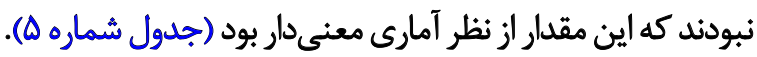
نمودار بقاى كايلان ماير در تصوير شماره ا نشان داده شده
در سالمندانى كه بيمارى زمينهاي نداشتئد، به طور معنى الرارى

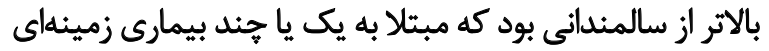

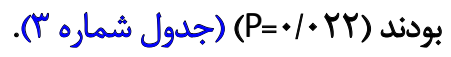
در خصوص ييامدهاى اندازمكيرىشده در سالمندان مبتلا به

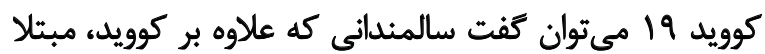

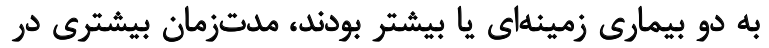

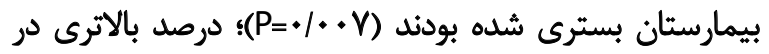

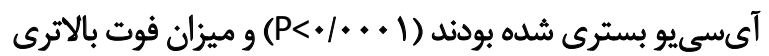

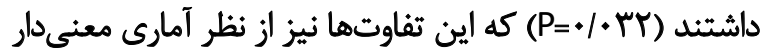
بودند (جدول شماره (f). نتايج رگرسيون لجستيك نشان داد داشتن بيمارىهاى

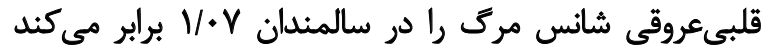

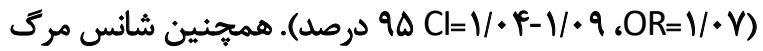

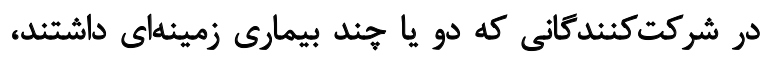

جدول هـ ـ نسبت شانس بيمارىهاى زمينهاى به تفكيك هر بيمارى و تركيب بيمارىهاى زميئهاى براى ايجاد مركى يا بسترى در بيمارستان

\begin{tabular}{|c|c|c|c|c|c|}
\hline \multicolumn{2}{|c|}{ نسبت شانس براى بيامد بسترى در آى سىيو } & \multicolumn{2}{|c|}{ نسبت شانس براى ييامد مرئ } & \multirow[b]{2}{*}{ متغيرها } & \\
\hline $\mathbf{P}$ & نسبت شانس (OR) و فاصينان (CI) & $\mathbf{P}$ & نسبت ثشانس (OR) و فاصينان (CI) & & \\
\hline $.1 \cdot m$ & $V / A F(V / \cdot \Delta-Y / \Delta \Delta)$ & $.1 \ldots+1$ & $1 / \cdot y(1 /+4-1 /+9)$ & بيمارى هاى قلبى عروقى & \multirow{8}{*}{ يمارى باى زمينهاي } \\
\hline$\cdot / P r$ & $V / M T(\cdot / 81-Y / A Y)$ & $\cdot / r+r$ & $\cdot / r(\cdot / \pi f-1 / \Delta \Delta)$ & ييمارى هاي ثنفسى & \\
\hline . /rra & $W \cdot(\cdot M-+* \cdot \Delta)$ &.$|8 \Delta|$ & $M / r m(\cdot / R \wedge-r / N r)$ & بيمارىهاى كليوى & \\
\hline $.1 \cdot 18$ & $1 / 41(\cdot / Q \Delta-r / 1 \cdot)$ &.$/ m w^{\prime \prime}$ & WrI $(\cdot M q-V / A F)$ & فشارخون & \\
\hline $.1 \cdot 1$ & $M / Y \cdot(Y / / Y-Y / \Delta S)$ & . & $M T \cdot(\cdot \mid A \Delta-r / \cdot 1)$ & ديابت & \\
\hline $.1 \cdot 1 V$ & $r / / r(Y / / f-r / q V)$ & $\cdot / \mathrm{VA}$ & $\cdot / 9 \cdot(\cdot / 90-\mid / A 1)$ & سكته مغزى & \\
\hline .1018 & $V / * \lambda(* / F a-r / A r)$ & - IAST & $\cdot / 19(+/ \pi r-r / r N)$ & بيمارىهاى تيروئيلى & \\
\hline \multirow[t]{2}{*}{$\cdot / N \cdot 9$} & $1 / T \&(\cdot / r q-F / F I)$ & ./Ne & $T / N F(\cdot / N \Delta-N M T)$ & بيمارى هاى رماتيسمى & \\
\hline & 1 & & 1 & بلدون بيمارى زمينهاى & \multirow{3}{*}{ تعداد بيمارىهاى زميئهاى } \\
\hline$\cdot / \cdot V$ & $1 / 8 \Lambda(+/ 9 \Delta-Y / 9 \Delta)$ & $+/ 199$ & $1 / \uparrow \&(+/ \Lambda I Y-Y / \& Y)$ & 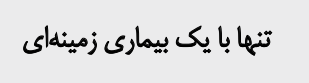 & \\
\hline$\%+r$ & $r / T E(I / M E-r / A I)$ & $+1+4$ & $1 / 89(+/ 97-r / q 1)$ & با دو بيمارى زمينهاى يا بيشتر & \\
\hline
\end{tabular}




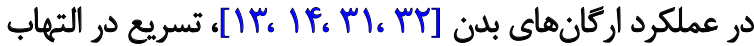

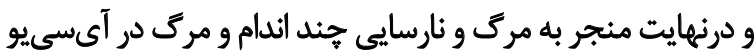

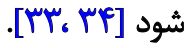

در مطالعات بيشين نشان داده شده است كه همزمانى ابتلا

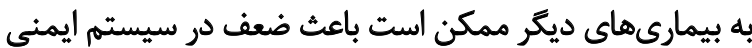

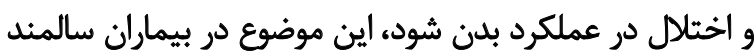

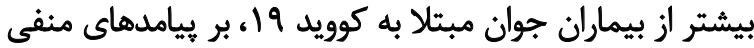

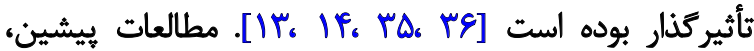

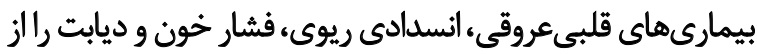

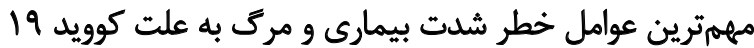

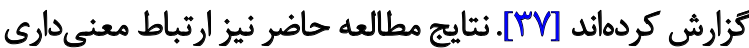

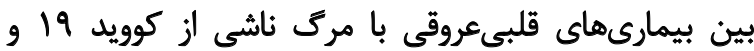

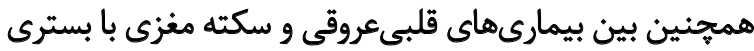

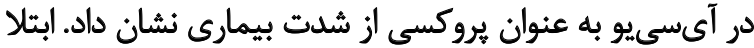

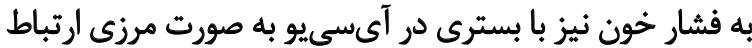

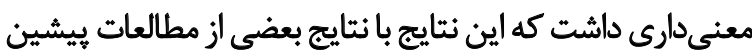

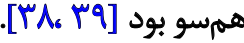

در خصوص ارتباط بيامدهاى منفى كوويد 19 و همرابتلايى

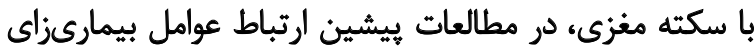

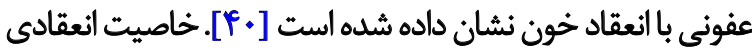

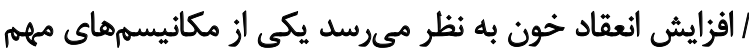

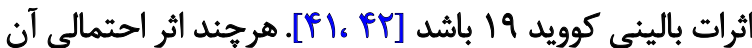
در علت سكته مغزى هنوز

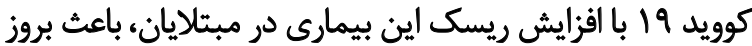

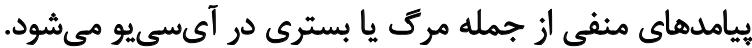

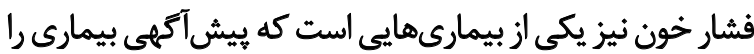

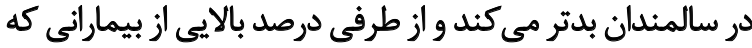

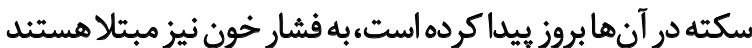

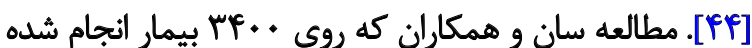

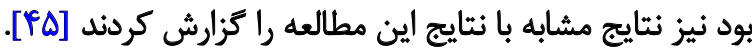

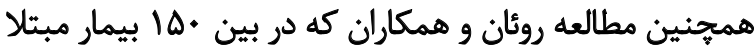

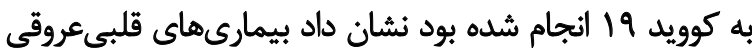

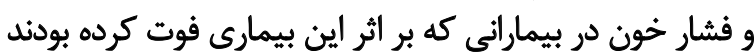

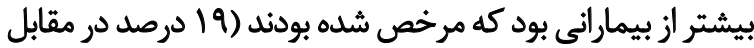

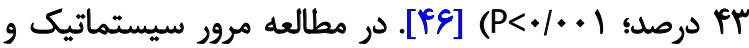

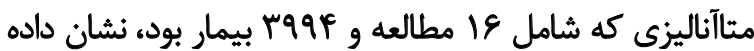

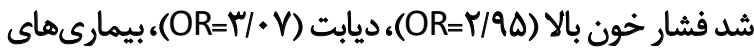

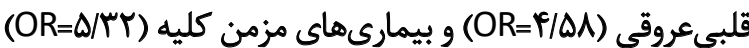

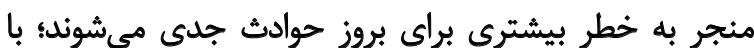

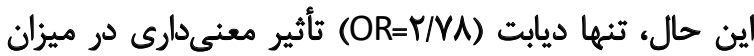

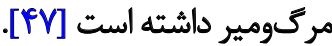

ارتباط بين ديابت و ييامدهاي منفى كوويد 9 انيز در مطالعات

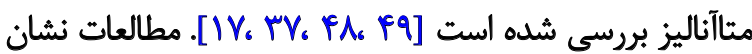

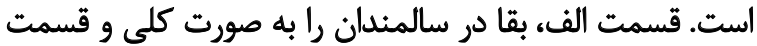

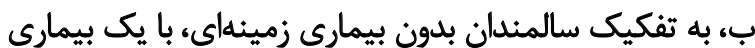

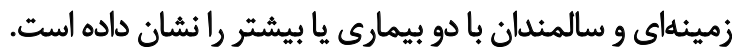

در تصوير شماره Y نيز ارتباط بين ييامدهاي اصلى كوويد 19 با

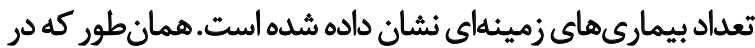

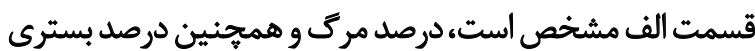

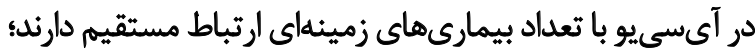

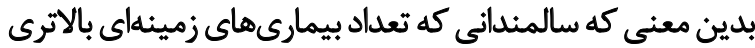

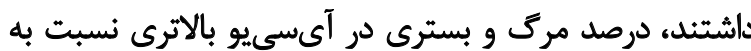

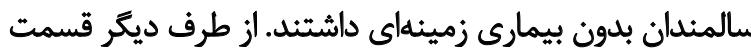

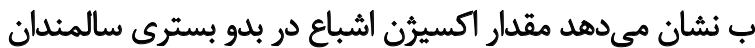

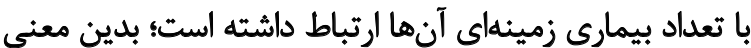

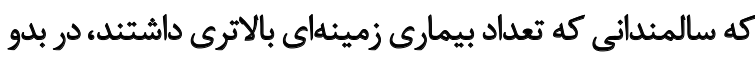
بسترى، مقدار اكسيرن اشباع پايينترى داشئن داشتند.

بحث

بيمارى كوويد 19 باعث إيجاد يك غاندمى شديد شداده كه

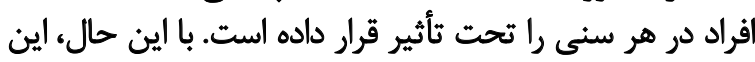

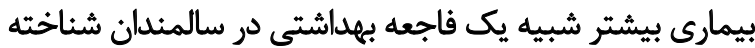

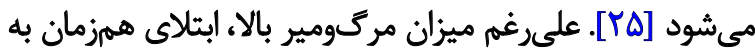

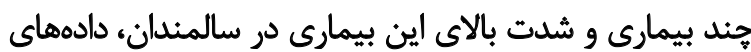

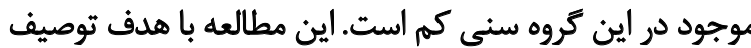

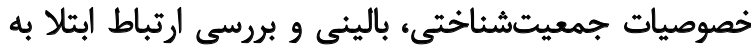

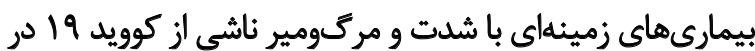

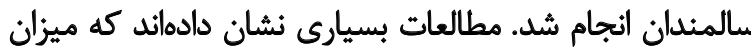

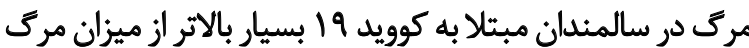

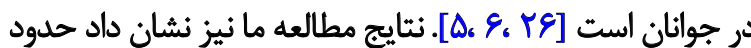

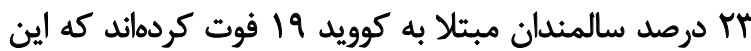
مقدار از ميزان كشندگى در سالمندان جيني، كره و و همجينين

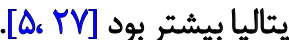

بيمارىهاى مزمن به يك بار اقتصادى جهانى تبديل شدهاند

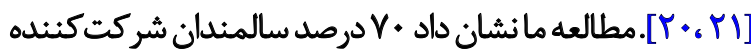

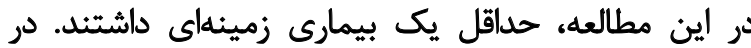

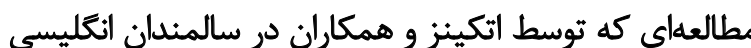

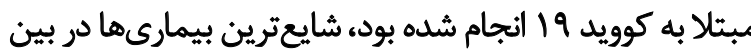

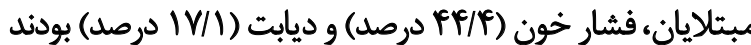

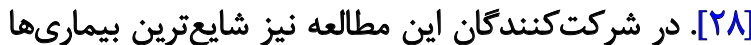

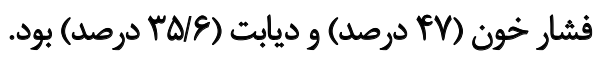
بيماران مبتلابه كوويد 9 ا معمولاً به دلايل مختلفى مثل نارسايى تاريى

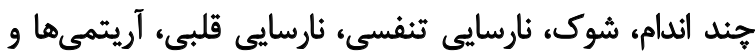

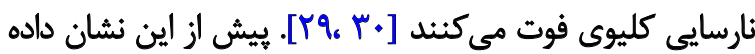

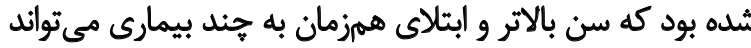

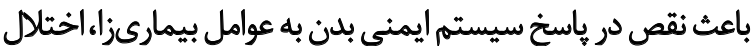


بهار +.1F. بهوره 19. شماره

ill

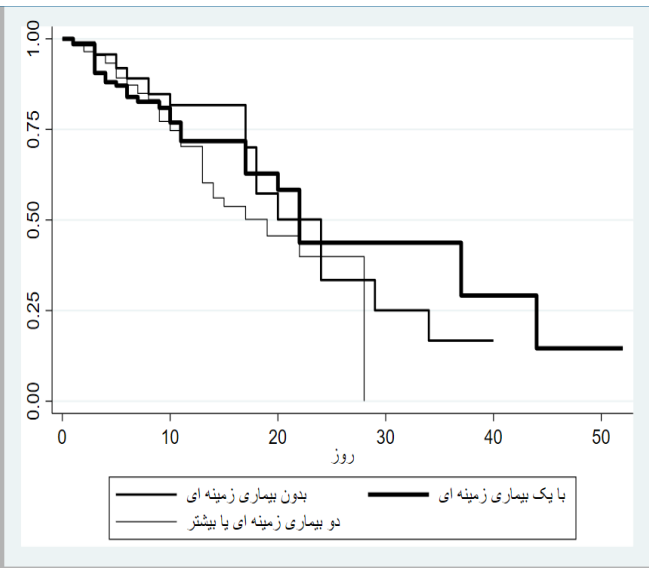

ب

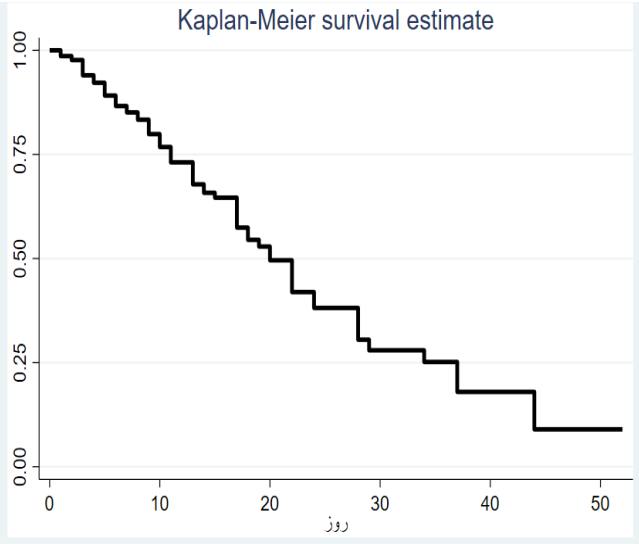

الف

تصوير ا. نمودار احتمال بقاى كايلان ماير در افراد سالمند مورد مطالعه. الف: دركل افراد، ب: به تفكيك بيمارىهاي زمينهاى

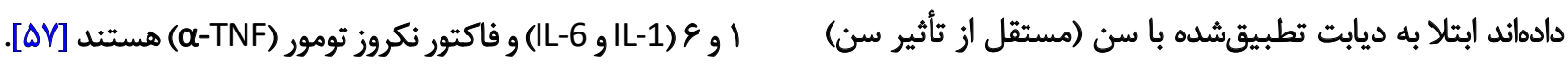
نشائكر هاى مختلفي از جمله C-reactive protien، فيبرينورثن

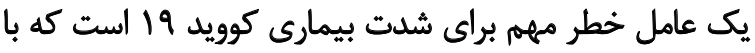

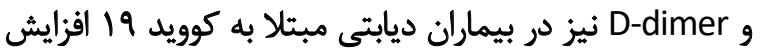

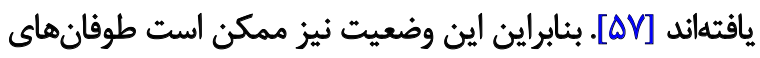

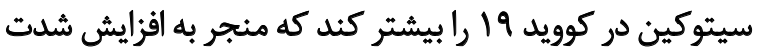

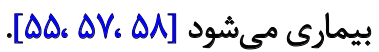

بيمارىهاي قلبي عروقي يكي از شايعترين بيمارىهاي مزمن

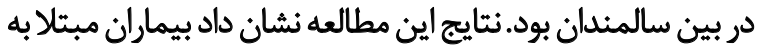

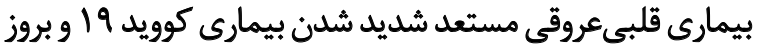

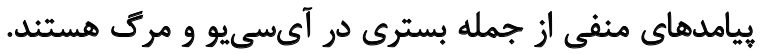

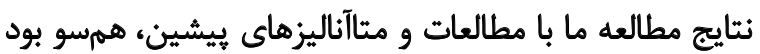

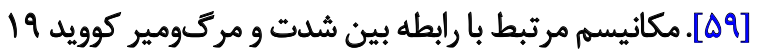

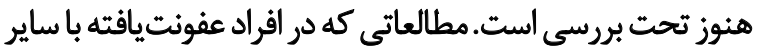

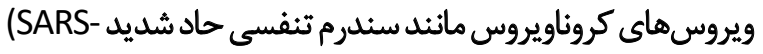
و سندرم تنفسى خاورميانه CoV)

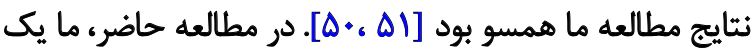

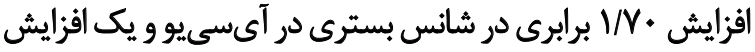

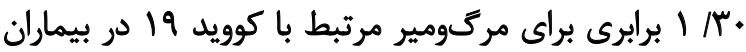

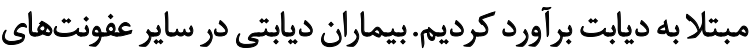

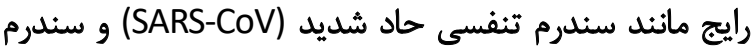

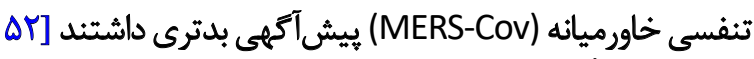

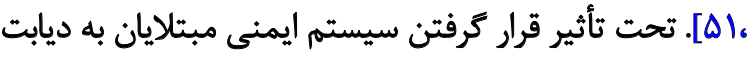

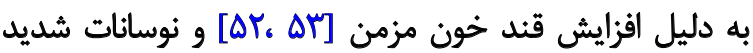

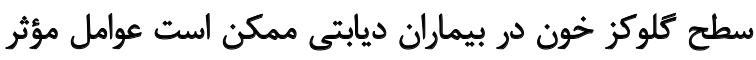

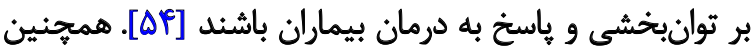

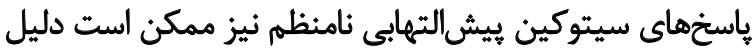

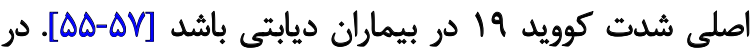

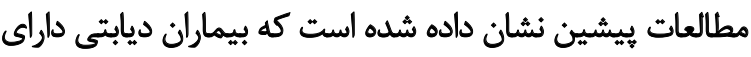

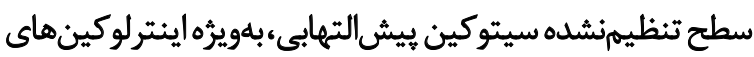
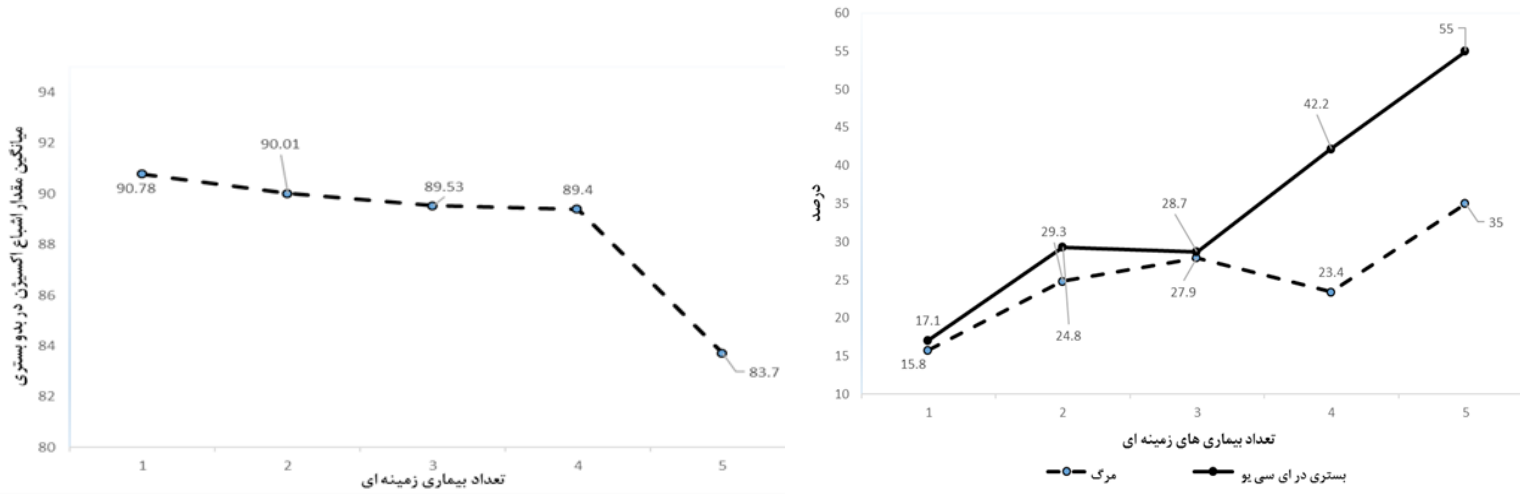

الف

تصوير Y. افزايش بيامدهاي اصلي بيمارى با افزايش تعداد بيمارىهاي زميئهاي در سالمثدان بسترى شده 记 الف: درصد بسترى در آىسىيو، مركى؛ ب: ميائكين اكسيرن.

9v 


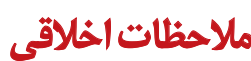 \\ يبيروى از اصول اخلاق يروهش}

ضوابط اخلاقى اين طرح در دانشكاه علوميزشكى تهران مورد

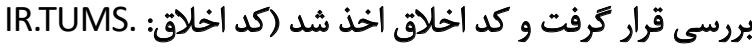

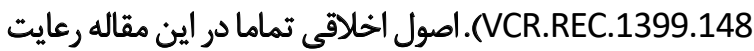

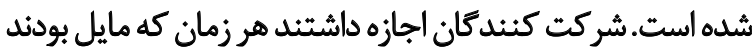

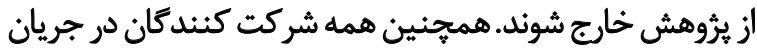

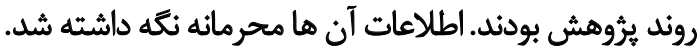

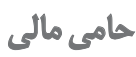

اين يروهش با كمك مالى دانشكاه علوميزشكى تهران انجام

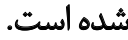

\section{مشاركت نويسندكَان}

تحقيق و بررسى متون: حامد اخوىزادكان و محمدقاسم

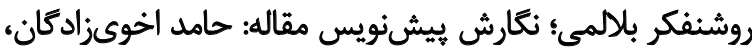

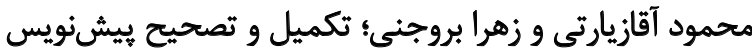

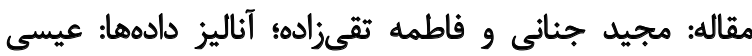
اكبرزاده عرب و مجيد جنانى؛ ويراستارى، تكميل و تأييد نهايى:

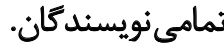

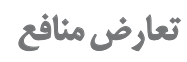

بنابر اظهار نويسندكان اين مقاله تعارض منافع ندارد.

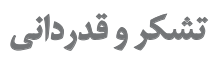

ازئ يرسنل و مديريت محترم بيمارستان بهارلو كه بامادر اجرائ اين طرح همكارى كردند، كمال تشكر و قدردانى را داريم.
بودند، افزايش خطر مركومير در بيماران مبتلا به بيمارىهاى

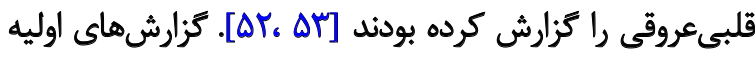

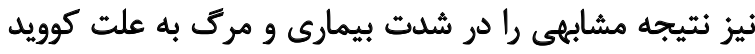

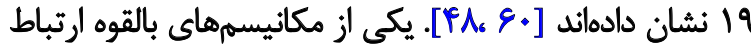

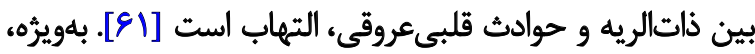

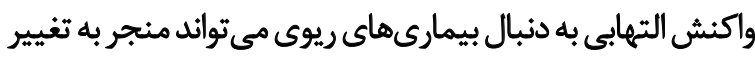

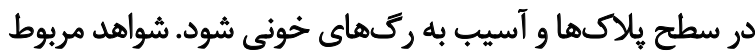

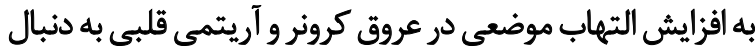

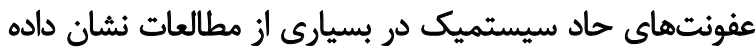

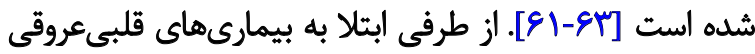

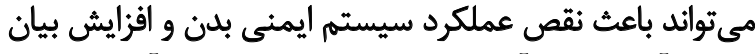

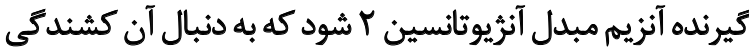

يا شدت بيمارى كوويد 19 افزايش مى بيابد [9F].

\section{نتيجلكيرى نههايى}

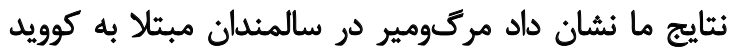

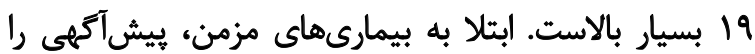

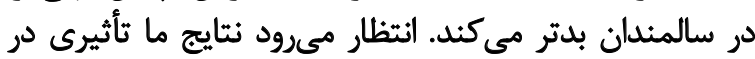

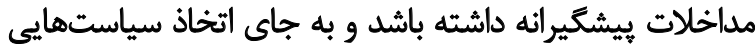

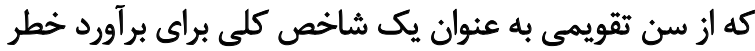

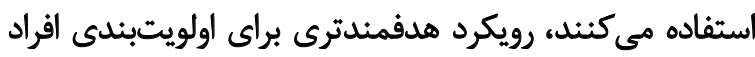

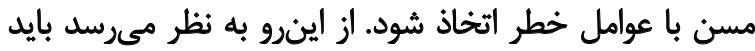

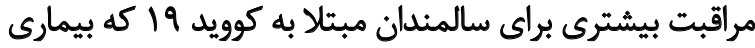

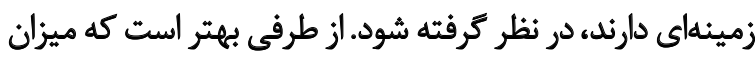

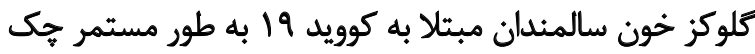

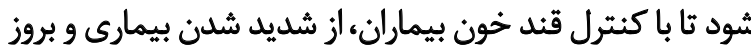
مرك بيشكيرى به عمل آيد.

يكى از محدوديتهاى اين مطالعه اين است كه تعداد بيامدها

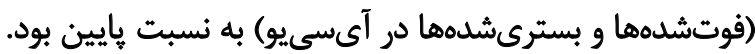

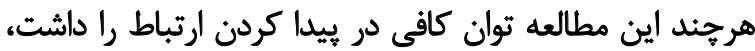

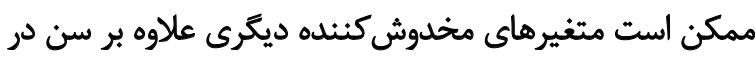

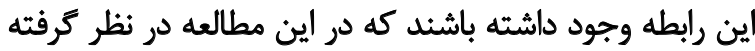

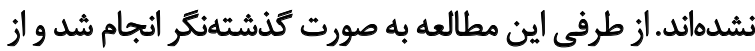

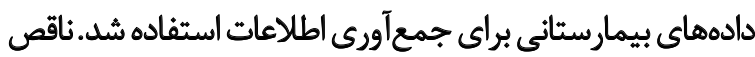

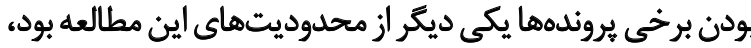

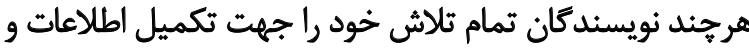
برس از بيماران انجام دادند.

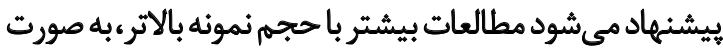

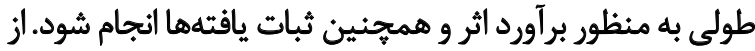

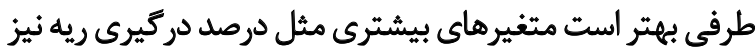
اندازهكيرى و اثر آن تطبيق داده شود. 


\section{Reference}

[1] Huang C, Wang Y, Li X, Ren L, Zhao J, Hu Y, et al. Clinical features of patients infected with 2019 novel coronavirus in Wuhan, China. The Lancet. 2020;395(10223):497-506. [DOI:10.1016/S01406736(20)30183-5]

[2] Worldometers. COVID-19 coronavirus pandemic. 2021 [Updated 2021 January 24, 2021]. Available from: https://www.worldometers.info/coronavirus/

[3] Cucinotta D, Vanelli M. WHO declares COVID-19 a pandemic. Acta Bio-Medica: Atenei Parmensis. 2020; 91(1):157-60. [doi: 10.23750/abm.v91i1.9397]

[4] Meyerowitz-Katz G, Merone L. A systematic review and meta-analysis of published research data on COVID-19 infectionfatality rates. International Journal of Infectious Diseases. 2020; 101:138-48. [DOI:10.1101/2020.05.03.20089854]

[5] Kang SJ, Jung SI. Age-related morbidity and mortality among patients with COVID-19. Infection \& Chemotherapy. 2020; 52(2):154-64. [DOI:10.3947/ic.2020.52.2.154] [PMID] [PMCID]

[6] Leung C. Risk factors for predicting mortality in elderly patients with COVID-19: A review of clinical data in China. Mechanisms of Ageing and Development. 2020; 188:111255. [DOI:10.1016/j. mad.2020.111255] [PMID] [PMCID]

[7] Parohan M, Yaghoubi S, Seraji A, Javanbakht MH, Sarraf P, Djalali M. Risk factors for mortality in patients with Coronavirus disease 2019 (COVID-19) infection: A systematic review and meta-analysis of observational studies. The Aging Male. 2020; 23(5):1416-24. [DOI:10.1101/2020.04.09.20056291]

[8] Hussain A, Mahawar K, Xia Z, Yang W, Shamsi E-H. Obesity and mortality of COVID-19. Meta-analysis. Obesity Research \& Clinical Practice. 2020; 14(4):295-300. [DOI:10.1016/j.orcp.2020.07.002] [PMID] [PMCID]

[9] Pinto LC, Bertoluci MC. Type 2 diabetes as a major risk factor for COVID-19 severity: A meta-analysis. Archives of Endocrinology and Metabolism. 2020; 64(3):199-200. [DOI:10.20945/23593997000000256] [PMID]

[10] Ji Y, Ma Z, Peppelenbosch MP, Pan Q. Potential association between COVID-19 mortality and health-care resource availability. The Lancet Global Health. 2020; 8(4):e480. [DOI:10.1016/S2214109X(20)30068-1]

[11] Ou M, Zhu J, Ji P, Li H, Zhong Z, Li B, et al. Risk factors of severe cases with COVID-19: A meta-analysis. Epidemiology \& Infection. 2020; 148:e175. [DOI:10.1017/S095026882000179X] [PMID] [PMCID]

[12] Rahman A, Sathi NJ. Risk factors of the severity of COVID_19: A meta_analysis. International Journal of Clinical Practice. 2020; e13916. [DOI:10.1111/ijcp.13916]

[13] Lara PC, Macías-Verde D, Burgos-Burgos J. Age-induced NLRP3 Inflammasome over-activation increases lethality of SARS-CoV-2 pneumonia in elderly patients. Aging and Disease. 2020; 11(4):756-62. [DOI:10.14336/AD.2020.0601] [PMID] [PMCID]

[14] Dorshkind K, Swain S. Age-associated declines in immune system development and function: causes, consequences, and reversal. Current Opinion in Immunology. 2009; 21(4):404-7. [DOI:10.1016/j.coi.2009.07.001] [PMID] [PMCID]
[15] Zhou X, Chen D, Wang L, Zhao Y, Wei L, Chen Z, et al. Low serum calcium: A new, important indicator of COVID-19 patients from mild/moderate to severe/critical. Bioscience Reports. 2020; 40(12):BSR20202690. [DOI:10.1042/BSR20202690] [PMID] [PMCID]

[16] Sun JK, Zhang WH, Zou L, Liu Y, Li JJ, Kan XH, et al. Serum calcium as a biomarker of clinical severity and prognosis in patients with coronavirus disease 2019. Aging. 2020 12(12):11287-95. [DOI:10.18632/aging.103526] [PMID] [PMCID]

[17] Wang B, Li R, Lu Z, Huang Y. Does comorbidity increase the risk of patients with COVID-19: Evidence from meta-analysis. Aging. 2020; 12(7):6049-57. [DOI:10.18632/aging.103000] [PMID] [PMCID]

[18] Fang X, Li S, Yu H, Wang P, Zhang Y, Chen Z, et al. Epidemiological, comorbidity factors with severity and prognosis of COVID-19: A systematic review and meta-analysis. Aging. 2020; 12(13):12493-503. [DOI:10.18632/aging.103579] [PMID] [PMCID]

[19] Norman GR, Stratford P, Regehr G. Methodological problems in the retrospective computation of responsiveness to change: The lesson of Cronbach. Journal of Clinical Epidemiology. 1997; 50(8):869-79. [DOI:10.1016/S0895-4356(97)00097-8]

[20] Wolff JL, Starfield B, Anderson G. Prevalence, expenditures, and complications of multiple chronic conditions in the elderly. Archives of Internal Medicine. 2002; 162(20):2269-76. [DOI:10.1001/archinte.162.20.2269] [PMID]

[21] Fortin M, Bravo G, Hudon C, Vanasse A, Lapointe L. Prevalence of multimorbidity among adults seen in family practice. The Annals of Family Medicine. 2005; 3(3):223-8. [DOI:10.1370/ afm.272] [PMID] [PMCID]

[22] Richardson S, Hirsch JS, Narasimhan M, Crawford JM, McGinn T, Davidson KW, et al. Presenting characteristics, comorbidities, and outcomes among 5700 patients hospitalized with COVID-19 in the New York City area. JAMA. 2020; 323(20):2052-9. [doi: 10.1001/jama.2020.6775]

[23] Fortin M, Lapointe L, Hudon C, Vanasse A. Multimorbidity is common to family practice: Is it commonly researched? Canadian Family Physician. 2005; 51(2):244-5. [PMCID]

[24] Gonzalez JS, SafrenSA, Cagliero E, Wexler DJ, Delahanty L, Wittenberg E, et al. Depression, self-care, and medication adherence in type 2 diabetes: Relationships across the full range of symptom severity. Diabetes Care. 2007; 30(9):2222-7. [DOI:10.2337/dc070158] [PMID] [PMCID]

[25] Salimi S, Hamlyn JM. COVID-19 and crosstalk with the hallmarks of aging. The Journals of Gerontology: Series A. 2020; 75(9):e34-41. [DOI:10.1093/gerona/glaa149] [PMID] [PMCID]

[26] Martín-Sánchez FJ, Del Toro E, Cardassay E, Carbó AV, Cuesta $\mathrm{F}$, Vigara $\mathrm{M}$, et al. Clinical presentation and outcome across age categories among patients with COVID-19 admitted to a Spanish Emergency Department. European Geriatric Medicine. 2020 11(5):829-41. [DOI:10.1007/s41999-020-00359-2] [PMID] [PMCID]

[27] Lee JY, Kim HA, Huh K, Hyun M, Rhee J-Y, Jang S, et al. Risk factors for mortality and respiratory support in elderly patients hospitalized with COVID-19 in Korea. Journal of Korean Medical Science. 2020; 35(23):e223. [DOI:10.3346/jkms.2020.35.e223] [PMID] [PMCID]

[28] Atkins J, Masoli J, Delgado J, Pilling L, Kuo C, Kuchel G, et al Preexisting comorbidities predicting severe COVID-19 in older adults in the UK biobank community cohort. The Journals of Gerontology: Series A. 2020; 75(11):2224-30. [DOI:10.1101/2020.05.0 6.20092700] 
[29] Novel CPERE. [The epidemiological characteristics of an outbreak of 2019 novel coronavirus diseases (COVID-19) in China (Chinese)]. Zhonghua Liu Xing Bing Xue Za Zhi = Zhonghua Liuxingbingxue Zazhi. 2020; 41(2):145-51. [DOI:10.46234/ccdcw2020.032]

[30] Chen N, Zhou M, Dong X, Qu J, Gong F, Han Y, et al. Epidemiological and clinical characteristics of 99 cases of 2019 novel coronavirus pneumonia in Wuhan, China: A descriptive study. The Lancet. 2020; 395(10223):507-13. [DOI:10.1016/S01406736(20)30211-7]

[31] Tabas I, Glass CK. Anti-inflammatory therapy in chronic disease: Challenges and opportunities. Science. 2013, 339(6116):166-72. [DOI:10.1126/science.1230720] [PMID] [PMCID]

[32] Fernández-Ruiz I. Immune system and cardiovascular disease. Nature Reviews Cardiology. 2016; 13:503 [DOI:10.1038/s41569018-0056-2]

[33] Ragab D, Salah Eldin H, Taeimah M, Khattab R, Salem R. The COVID-19 cytokine storm; What we know so far. Frontiers in Immunology. 2020; 11:1446. [DOI:10.3389/fimmu.2020.01446] [PMID] [PMCID]

[34] Tran DD, Groeneveld A, Van der Meulen J, Nauta J, Strack van Schijndel R, Thijs L. Age, chronic disease, sepsis, organ system failure, and mortality in a medical intensive care unit. Critical Care Medicine. 1990; 18(5):474-9. [DOI:10.1097/00003246199005000-00002] [PMID]

[35] Sanyaolu A, Okorie C, Marinkovic A, Patidar R, Younis K, Desai $\mathrm{P}$, et al. Comorbidity and its Impact on Patients with COVID-19. SN Comprehensive Clinical Medicine. 2020; 2:1069-76. [DOI:10.1007/s42399-020-00363-4] [PMID] [PMCID]

[36] Iaccarino G, Grassi G, Borghi C, Ferri C, Salvetti M, Volpe M. Age and multimorbidity predict death among COVID-19 patients: Results of the SARS-RAS study of the Italian Society of Hypertension. Hypertension. 2020; 76(2):366-72. [DOI:10.1161/ HYPERTENSIONAHA.120.15324] [PMID]

[37] de Almeida-Pititto B, Dualib PM, Zajdenverg L, Dantas JR, De Souza FD, Rodacki M, et al. Severity and mortality of COVID 19 in patients with diabetes, hypertension and cardiovascular disease: A meta-analysis. Diabetology \& Metabolic Syndrome. 2020 12:75. [DOI:10.1186/s13098-020-00586-4] [PMID] [PMCID]

[38] Fuchs L, Novack V, McLennan S, Celi LA, Baumfeld Y, Park S, et al. Trends in severity of illness on ICU admission and mortality among the elderly. PLoS One. 2014; 9(4):e93234. [DOI:10.1371/ journal.pone.0093234] [PMID] [PMCID]

[39] Cook T. The importance of hypertension as a risk factor for severe illness and mortality in COVID_19. Anaesthesia. 2020; 75(7):976-7. [DOI:10.1111/anae.15103] [PMID] [PMCID]

[40] Jillella DV, Wisco DR. Infectious causes of stroke. Current Opinion in Infectious Diseases. 2019; 32(3):285-92. [DOI:10.1097/ QCO.0000000000000547] [PMID]

[41] Spiezia L, Boscolo A, Poletto F, Cerruti L, Tiberio I, Campello $\mathrm{E}$, et al. COVID-19-related severe hypercoagulability in patients admitted to intensive care unit for acute respiratory failure. Thrombosis and Haemostasis. 2020; 120(6):998-1000 [DOI:10.1055/s-0040-1710018] [PMID] [PMCID]

[42] Panigada M, Bottino N, Tagliabue P, Grasselli G, Novembrino C, Chantarangkul V, et al. Hypercoagulability of COVID_19 patients in intensive care unit: A report of thromboelastography findings and other parameters of hemostasis. Journal of Thrombosis and Haemostasis. 2020; 18(7):1738-42. [DOI:10.1111/ jth.14850] [PMID]
[43] Oxley TJ, Mocco J, Majidi S, Kellner CP, Shoirah H, Singh IP, et al. Large-vessel stroke as a presenting feature of Covid-19 in the young. New England Journal of Medicine. 2020; 382(20):e60. [DOI:10.1056/NEJMc2009787] [PMID] [PMCID]

[44] Singh R, Suh I, Singh V, Chaithiraphan S, Laothavorn P, Sy R, et al. Hypertension and stroke in Asia: Prevalence, control and strategies in developing countries for prevention. Journal of Human Hypertension. 2000; 14(10):749-63. [DOI:10.1038/si.jhh.1001057] [PMID]

[45] Sun Y, Guan X, Jia L, Xing N, Cheng L, Liu B, et al. Independent and combined effects of hypertension and diabetes on clinical outcomes in patients with COVID_19: A retrospective cohort study of Huoshen Mountain Hospital and Guanggu Fangcang Shelter Hospital. The Journal of Clinical Hypertension. 2021 23(2):218-31. [DOI:10.1111/jch.14146] [PMID]

[46] Ruan Q, Yang K, Wang W, Jiang L, Song J. Clinical predictors of mortality due to COVID-19 based on an analysis of data of 150 patients from Wuhan, China. Intensive Care Medicine. 2020; 46(5):846-8. [DOI:10.1007/s00134-020-05991-x] [PMID] [PMCID]

[47] Nandy K, Salunke A, Pathak SK, Pandey A, Doctor C, Pu $\mathrm{K}$, et al. Coronavirus disease (COVID-19): A systematic review and meta-analysis to evaluate the impact of various comorbidities on serious events. Diabetes \& Metabolic Syndrome. 2020 14(5):1017-25. [DOI:10.1016/j.dsx.2020.06.064] [PMID] [PMCID]

[48] Li B, Yang J, Zhao F, Zhi L, Wang X, Liu L, et al. Prevalence and impact of cardiovascular metabolic diseases on COVID-19 in China. Clinical Research in Cardiology. 2020; 109(5):531-8 [DOI:10.1007/s00392-020-01626-9] [PMID] [PMCID]

[49] Kumar A, Arora A, Sharma P, Anikhindi SA, Bansal N, Singla $\mathrm{V}$, et al. Is diabetes mellitus associated with mortality and severity of COVID-19? A meta-analysis. Diabetes \& Metabolic Syndrome Clinical Research \& Reviews. 2020; 14(4):535-45. [DOI:10.1016/j. dsx.2020.04.044] [PMID] [PMCID]

[50] Huang I, Lim MA, Pranata R. Diabetes mellitus is associated with increased mortality and severity of disease in COVID-19 pneumonia-a systematic review, meta-analysis, and meta-regression. Diabetes \& Metabolic Syndrome. 2020; 14(4):395-403. [DOI:10.1016/j.dsx.2020.04.018] [PMID] [PMCID]

[51] Palaiodimos L, Chamorro-Pareja N, Karamanis D, Li W, Zavras PD, Chang KM, et al. Diabetes is associated with increased risk for in-hospital mortality in patients with COVID-19: A systematic review and meta-analysis comprising 18,506 patients. Hormones 2021; 20(2):305-14. [DOI:10.1007/s42000-020-00246-2] [PMID] [PMCID]

[52] Morra ME, Van Thanh L, Kamel MG, Ghazy AA, Altibi AM Dat LM, et al. Clinical outcomes of current medical approaches for Middle East respiratory syndrome: A systematic review and meta_analysis. Reviews in Medical Virology. 2018; 28(3):e1977. [DOI:10.1002/rmv.1977] [PMID] [PMCID]

[53] Chan JWM, Ng CK, Chan YH, Mok TYW, Lee S, Chu SYY, et al. Short term outcome and risk factors for adverse clinical outcomes in adults with severe acute respiratory syndrome (SARS). Thorax 2003; 58(8):686-9. [DOI:10.1136/thorax.58.8.686] [PMID] [PMCID]

[54] Chen T, Wu D, Chen H, Yan W, Yang D, Chen G. Clinical characteristics of 113 deceased patients with coronavirus disease 2019 Retrospective study. BMJ. 2020; 368:m1091. [doi: 10.1136/bmj. m1091.] 
[55] Ceriello A. Management of diabetes today: An exciting confusion. Diabetes Research and Clinical Practice. 2020; 162:108129[DOI:10.1016/j.diabres.2020.108129] [PMID]

[56] Maddaloni E, Buzzetti R. Covid_19 and diabetes mellitus: Unveiling the interaction of two pandemics. Diabetes/Metabolism Research and Reviews. 2020; 36(7):e33213321. [DOI:10.1002/ dmrr.3321] [PMCID]

[57] Geerlings SE, Hoepelman AI. Immune dysfunction in patients with diabetes mellitus (DM). FEMS Immunology \& Medical Microbiology. 1999; 26(3-4):259-65. [DOI:10.1111/j.1574-695X.1999. tb01397.x] [PMID]

[58] Mehta P, McAuley DF, Brown M, Sanchez E, Tattersall RS, Manson JJ. COVID-19: Consider cytokine storm syndromes and immunosuppression. The Lancet. 2020; 395(10229):1033-4. [DOI:10.1016/S0140-6736(20)30628-0]

[59] Tian W, Jiang W, Yao J, Nicholson CJ, Li RH, Sigurslid HH, et al. Predictors of mortality in hospitalized COVID_19 patients: A systematic review and meta_analysis. Journal of Medical Virology. 2020; 92(10):1875-83. [DOI:10.1002/jmv.26050] [PMID] [PMCID]

[60] Wang X, Fang X, Cai Z, Wu X, Gao X, Min J, et al. Comorbid chronic diseases and acute organ injuries are strongly correlated with disease severity and mortality among COVID-19 patients: A systemic review and meta-analysis. Research. 2020; 2020:2402961. [DOI:10.34133/2020/2402961] [PMID] [PMCID]

[61] Libby P. Mechanisms of acute coronary syndromes and their implications for therapy. The New England Journal of Medicine. 2013; 368(21):2004-13. [DOI:10.1056/NEJMra1216063] [PMID]

[62] Madjid M, Vela D, Khalili-Tabrizi H, Casscells SW, Litovsky S. Systemic infections cause exaggerated local inflammation in atherosclerotic coronary arteries: Clues to the triggering effect of acute infections on acute coronary syndromes. Texas Heart Institute Journal. 2007; 34(1):11-8. [PMCID]

[63] Mauriello A, Sangiorgi G, Fratoni S, Palmieri G, Bonanno E, Anemona $\mathrm{L}$, et al. Diffuse and active inflammation occurs in both vulnerable and stable plaques of the entire coronary tree: A histopathologic study of patients dying of acute myocardial infarction. Journal of the American College of Cardiology. 2005; 45(10):1585-93. [DOI:10.1016/j.jacc.2005.01.054] [PMID]

[64] Petrie JR, Guzik TJ, Touyz RM. Diabetes, hypertension, and cardiovascular disease: Clinical insights and vascular mechanisms. Canadian Journal of Cardiology. 2018; 34(5):575-84. [DOI:10.1016/j.cjca.2017.12.005] [PMID] [PMCID] 